\title{
Manipulation of photons in a cavity by dispersive atom-field coupling: Quantum-nondemolition measurements and generation of "Schrödinger cat" states
}

\author{
M. Brune, S. Haroche, and J. M. Raimond \\ Laboratoire de Spectroscopie Hertzienne de l'Ecole Normale Supérieure, 24 rue Lhomond, 75231 Paris CEDEX 05, France \\ L. Davidovich and N. Zagury \\ Departamento de Fisica, Pontificia Universidade Catolica, 22453 Rio de Janeiro, Brazil
}

(Received 1 November 1991)

\begin{abstract}
A quantum-nondemolition method to measure the number of photons stored in a high- $Q$ cavity, introduced by Brune et al. [Phys. Rev. Lett. 65, 976 (1990)], is described in detail. It is based on the detection of the dispersive phase shift produced by the field on the wave function of nonresonant atoms crossing the cavity. This shift can be measured by atomic interferometry, using the Ramsey separatedoscillatory-field method. The information acquired by detecting a sequence of atoms modifies the field step by step, until it eventually collapses into a Fock state. At the same time, the field phase undergoes a diffusive process as a result of the back action of the measurement on the photon-number conjugate variable. Once a Fock state has been generated, its evolution under weak perturbation can be continuously monitored, revealing quantum jumps between various photon numbers. When applied to an initial coherent field, the intermediate steps of the measuring sequence produce quantum superpositions of classical fields, known as "Schrödinger cat states." Ways to prepare and detect these states in a cavity subjected to a weak relaxation process are discussed. The effects analyzed in this article could realistically be observed by using circular Rydberg atoms and very high- $Q$ superconducting microwave cavities. The possibility of photon "manipulation" through nonresonant atom-field interactions opens a domain in cavity QED studies.
\end{abstract}

PACS number(s): 42.50.Dv, 03.65.Bz

\section{INTRODUCTION}

The detection of light intensity by usual photocounting techniques is field destructive. Successive measurements of the intensity yield different results, as the initial field is depleted by the continuing measuring process. Methods to avoid the "back action" of the measurement on the detected observable have been proposed $[1,2]$ and implemented in the optical domain [3]. These experiments are the realization of the "quantum-nondemolition" (QND) schemes introduced in [4]. They rely on nonlinear coupling of the signal field to be measured with a probe field whose phase is altered by a quantity depending on the number of photons in the signal beam. The coupling is generally obtained via some kind of Kerr effect in a solid or gaseous medium. It requires a relatively intense signal beam containing a large number of photons. Moreover, the experiments have been performed so far on propagating beams, for which a continuous monitoring of the signal field is difficult to achieve.

We have recently proposed [5,6] a variant of these experiments in which the photons stored in a mode of a microwave resonant cavity are detected by measuring the dispersive phase shift experienced by the electric dipole of nonresonant Rydberg atoms sent one by one through the cavity. By implementing this quantumnondemolition scheme using a "dispersive atomic probe" (DAP-QND), fields with a very small number of photons could be continuously detected without back action on the photon number.
In the DAP-QND method, the measuring "meter" is a beam of two-level atoms crossing the cavity. Owing to the simplicity of this system, it is possible to perform realistic and complete simulations of the measurement, taking into account the quantum-mechanical randomness of each atomic detection process. These simulations show that the information on the field state is acquired progressively as successive atoms with random velocities cross the cavity and have their velocity and energy measured. Several such elementary atomic interaction and detection processes are required in order to perform a complete "measuring sequence," reducing the field into a Fock state. The collapse of the field into a pure photonnumber state thus appears as a multiple-atom event, entirely calculable, which can be followed in a single realization of a measuring sequence.

A very important characteristic of the DAP-QND method resides in the fact that each elementary process transforms the field state in a way a priori unpredictable, yet completely known once the atom has been detected. The interaction of the field with atoms followed by their detection thus generally constitutes a way to "manipulate" quantum fields without altering their energy. Fock states are not the only interesting nonclassical states which can be obtained in this way. It is also possible to prepare so-called "Schrödinger cat" states [7] of the field which are quantum superpositions of coherent states with macroscopically different phases or amplitudes [8]. These states can be obtained as a result of a single-atom interaction and detection process. 
An attractive feature of the DAP-QND method is to operate on fields "trapped" in a cavity, which remain accessible to observation for a long time, limited only by the system relaxation. In this respect, the experiments considered here are "dual" of those in which light beams are used to control, manipulate, and detect ions stored in a trap [9]. In our proposed DAP-QND scheme, the roles of field and matter are simply exchanged. Atoms are now used to manipulate and detect the field trapped in the cavity. Experiments recently performed in ion traps, such as the detection of quantum jumps [10] or the demonstration of the quantum Zeno effect [11], could be performed using the DAP-QND method on microwave fields stored in the cavity.

In this article, we present a comprehensive description of the DAP-QND scheme, which completes the qualitative analysis of [5]. We start with a review of QND methods in general, which allows us to introduce useful concepts and notations (Sec. II). The QND measurement of a microwave field using the detection of nonresonant radiative shifts produced on atoms crossing the cavity is described in Sec. III. We show that the atomic phase shifts can be conveniently measured by the Ramsey method of separated oscillatory fields [12], the cavity being sandwiched between two interaction zones where the atoms are subjected to a resonant coupling with an auxiliary microwave. Order-of-magnitude estimates on systems made of Rydberg atoms crossing superconducting cavities are presented, which show the feasibility of the method for measuring small fields down to the vacuum. The mechanism of the measurement and the collapse of the field into a Fock state are then analyzed in detail and simulations of experiments in which the number of photons in the cavity is continuously monitored are described (Sec. IV). The "back action" of the photon-number measurement, which affects the conjugate observable, i.e., the phase of the field, is then presented (Sec. V). We show that the field phase is perturbed essentially because each Rydberg atom introduced in the cavity acts as a "medium" with an index of refraction coupled to the mode during the atom-cavity crossing time. As the photon number gets reduced into a Fock state by successive interactions with nonresonant atoms, the phase of the field gets scrambled, providing a very direct illustration of phaseintensity complementarity.

A detailed analysis of the measuring sequence for a field initially prepared in a coherent state shows that the first interaction with an atom, followed by the atom detection, results in the preparation of a "Schrödinger cat" state, consisting in a superposition of two classical fields with different phases. The dephasing between the two components depends upon the atom velocity. Each component of this "cat" is "split" into two new states when the second atom is detected, and this process, which amounts to a phase diffusion, generally goes on until the phase is completely randomized. A notable exception to the phase randomization occurs when the dephasing produced by successive atoms is fixed and equal either to $\pi$ or to a simple rational multiple of $\pi$. This implies the use of monokinetic atoms, with a well-defined velocity. The field phase shift is then controlled and phase in- formation may be built and preserved into the field state, leading to the possibility of preparing and detecting stable "Schrödinger cat" states (Sec. VI).

Up to that point, the field mode will be described in the photon-number state representation. It is convenient, for simultaneous graphic descriptions of the field phase and amplitude and also for a simple description of field relaxation, to use the Wigner representation of the density matrix. In Sec. VII, we briefly translate the main results of this paper into this representation and we show how the Wigner distribution of a "Schrödinger cat" evolves under relaxation, in various circumstances. Finally, we conclude in Sec. VIII by discussing possible applications and generalizations of this method.

\section{REVIEW OF QND (REFS. [2] AND [13])}

The purpose of a QND method is to measure a signal observable $A_{S}$ of a quantum system $S$ (for example the energy or photon number of a field) by detecting a change in an observable $A_{P}$ of a probe $P$ coupled to $S$ during a finite measurement time $T$, without perturbing the subsequent evolution of $A_{S}$. The Hamiltonian of the $S+P$ system during $T$ can be written as

$$
H=H_{S}+H_{P}+H_{I},
$$

where $H_{S}, H_{P}$, and $H_{I}$ are, respectively, the Hamiltonians of $S, P$, and their mutual interaction. The $A_{i}$ observable ( $i=S$ or $P$ ) evolves according to

$$
i \hbar \frac{d A_{i}}{d t}=\left[A_{i}, H_{i}\right]+\left[A_{i}, H_{I}\right] \text {. }
$$

In order to use $P$ for a measurement of $A_{S}, H_{I}$ must obviously be a function of $A_{S}$ and the commutator of $A_{P}$ and $H_{I}$ must be different from zero:

$$
\begin{aligned}
& \frac{\partial H_{I}}{\partial A_{S}} \neq 0, \\
& {\left[A_{P}, H_{I}\right] \neq 0 .}
\end{aligned}
$$

Furthermore, $A_{S}$ should not be affected by its coupling to $A_{P}$ during the measurement:

$$
\left[A_{S}, H_{I}\right]=0 \text {. }
$$

After the $S-P$ interaction, the conjugate observable of $A_{S}, A_{S}^{c}$, is altered in an uncontrollable way ("back action" of the measurement). In order to avoid an uncontrollable perturbation on the subsequent evolution of $A_{S}, H_{S}$ should not be a function of $A_{S}^{c}$.

$$
\frac{\partial H_{S}}{\partial A_{S}^{c}}=0 \text {. }
$$

Conditions (3)-(6) define a QND measurement process [2,13]. Original QND ideas involved a dispersive coupling of the signal field to a material probe [4]. The idea has appeared unpractical, however, and QND schemes so far have used probe fields nonlinearly coupled to the signal via dispersive Kerr-type effects in a nonlinear solid medium or an atomic vapor [3]. Let us call $a_{S}, a_{S}^{\dagger}$ and 
$a_{P}, a_{P}^{\dagger}$ the annihilation and creation operators for the signal and probe fields (angular frequencies $\omega_{S}$ and $\omega_{P}$, respectively). In these schemes, a modification of the index of refraction of the medium, proportional to the number $A_{S}=a_{S}^{\dagger} a_{S}$ of signal photons, induces a phase shift of the probe. This shift produces in turn a change in the amplitude of a quadrature component of the probe:

$$
A_{P}=\left(a_{P}-a_{P}^{\dagger}\right) / 2 i \text {, }
$$

which can be measured by a homodyne method. The Hamiltonians $H_{i}(i=S$ or $P)$ are

$$
H_{i}=\hbar \omega_{i}\left(a_{i}^{\dagger} a_{i}+1 / 2\right)
$$

and the modified index of refraction corresponds to the existence of an effective interaction Hamiltonian $H_{I}$ between the signal and the probe, which can be expressed as

$$
H_{I}=\chi^{(3)} a_{P}^{\dagger} a_{P} a_{S}^{\dagger} a_{S},
$$

where $\chi^{(3)}$ is the Kerr nonlinear susceptibility of the medium. It is easy to verify that all the QND criteria are met. The amplitude of $A_{P}$ replicates the variations of the signal-field photon number without altering it. Physically, this is due to the fact that the interaction of the signal with the Kerr medium is nonresonant and thus cannot change the photon number. Of course the phase of the signal field (the $A_{S}^{c}$ observable in this case) is randomly altered by the measurement.

These QND methods are quite generally based on dispersive and nonlinear effects. The interaction $H_{I}$ must be at least quadratic in $a_{S}^{\dagger}$ and $a_{S}$ as opposed to the case of ordinary photodetection processes, where the interaction is linear in these operators. Usually, "highintensity" signal fields are required in order to induce appreciable nonlinearities in the medium. This is particularly the case for the schemes using solid materials as a Kerr medium.

\section{QND FIELD MEASUREMENTS BY DETECTION OF DISPERSIVE ATOMIC PHASE SHIFTS}

In the proposed DAP-QND method [5], the probe is no longer a field but a beam of atoms interacting nonlinearly and nonresonantly with the signal field. In order to achieve the large nonlinearities required for the method to be effective in detecting small photon numbers, the atoms are prepared into Rydberg states and the signal field is a microwave nearly resonant with a transition between adjacent Rydberg levels. Rydberg atoms coupled to small-size microwave cavities are indeed ideal systems to obtain strong couplings down to zero photons $[14,15]$.

\section{A. Radiative shifts of atoms coupled to the signal field}

Three Rydberg levels $e, i$, and $f$ play a role in the DAP-QND scheme. A possible configuration of these levels is shown in Fig. 1. Levels $i$ and $f$ (energies $E_{i}$ and $E_{f}$ ) are of the same parity, opposite to the parity of level $e$ (energy $E_{e}$ ). We assume, without loss of generality,

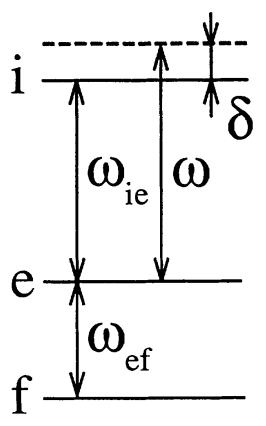

FIG. 1. Relevant Rydberg levels for the DAP-QND scheme.

that $E_{e}>E_{f}$. The cavity mode (angular frequency $\omega$ ) is slightly detuned from the $e \rightarrow i$ transition [frequency $\left.\omega_{i e}=\left(E_{i}-E_{e}\right) / \hbar\right]$. We call $\delta$ the frequency mismatch:

$$
\delta=\omega-\left|\omega_{i e}\right| \text {, }
$$

and $\Omega(\mathbf{r})$ the vacuum Rabi coupling $[15,16]$ between the atomic dipole on the $e \rightarrow i$ transition and the cavity mode. This coupling depends, through the field spatial distribution, upon the position $\mathbf{r}$ of the atom in the cavity. In the presence of $n$ photons, the coupling between states $e$ and $i$ is $\Omega \sqrt{n}$ (photon absorption) or $\Omega \sqrt{n+1}$ (photon emission). Let us assume that $\delta$ is large compared to $\Omega \sqrt{n}$ so that

$$
\Omega^{2} n / \delta^{2}<1
$$

On the other hand, $\delta$ is taken small compared to the difference in frequency between the $e \rightarrow i$ transition and all the other transitions in the Rydberg atom spectrum (notably the $e \rightarrow f$ one). Only levels $e$ and $i$ are then appreciably affected by the nonresonant atom-field coupling, which leaves level $f$ essentially unperturbed.

Let us consider the state $|e, n\rangle$ of the combined atomfield system, which represents the atom in level $e$ in the presence of $n$ photons. A simple perturbative analysis, valid if condition (11) is fulfilled, shows that $|e, n\rangle$, whose unperturbed energy is $E_{e}+(n+1 / 2) \hbar \omega$, experiences the energy shift

$$
\hbar \Delta_{e}(n, \mathbf{r})=\hbar \Omega^{2}(\mathbf{r}) n / \delta
$$

if $E_{e}<E_{i}$, and

$$
\hbar \Delta_{e}(n, \mathbf{r})=-\hbar \Omega^{2}(\mathbf{r})(n+1) / \delta
$$

if $E_{e}>E_{i}$.

The shifts described by Eq. (12) are quite similar to the usual "light shifts" observed in atomic systems interacting with nearly resonant light beams [17]. We consider here, however, the unusual situation in which a single photon [Eq. (12a)], or even the vacuum field [Eq. (12b)], is able to produce a measurable effect on the atomic levels. This is of course due to the large value of the vacuum Rabi coupling in Rydberg systems (see Sec. III C). For definiteness, we will consider in the following the $E_{e}<E_{i}$ case, corresponding to the energy-level configuration of 
Fig. 1, for which Eq. (12a) applies. The important point of this analysis is that the frequency of the $e \rightarrow f$ transition is shifted by an amount proportional to the number of signal photons.

\section{B. Using an atomic dipole quadrature as a QND probe}

Let us now focus on the subspace of the atomic Hilbert space spanned by the two states $e$ and $f$. The restriction $H_{\mathrm{at}}^{(e, f)}$ of the atomic Hamiltonian to this subspace can be expressed as

$$
H_{\mathrm{at}}^{(e, f)}=\hbar \omega_{e f} D_{e f}^{+} D_{e f}^{-},
$$

where $D_{e f}^{+}$and $D_{e f}^{-}$are "raising" and "lowering" atomic operators:

$$
D_{e f}^{+}=|e\rangle\left\langle f\left|, \quad D_{e f}^{-}=\left(D_{e f}^{+}\right)^{\dagger}=\right| f\right\rangle\langle e|
$$

(we choose here the energy of the $f$ state to be zero). In the presence of a cavity field satisfying condition (11), $H_{\text {at }}^{(e, f)}$ becomes an "effective" Hamiltonian:

$$
{ }^{\text {eff }} H_{\mathrm{at}}^{(e, f)}=\hbar\left[\omega_{e f}+\frac{\Omega^{2} n}{\delta}\right] D_{e f}^{+} D_{e f}^{-} .
$$

Consider now the atom (evolving in a superposition of levels $e$ and $f$ ) as the "probe" $P$ used to measure the signal-field photon number. Noticing that $n$ is the eigenvalue of the $a_{S}^{\dagger} a_{S}$ operator, we can express the effective $S-P$ coupling in this case as

$$
H_{I}=\frac{\hbar \Omega^{2}}{\delta} a_{S}^{\dagger} a_{S} D_{e f}^{+} D_{e f}^{-} .
$$

Let us define as probe observable the atomic dipole operator $A_{p}^{(\text {at })}$ :

$$
A_{P}^{(\text {at })}=\frac{1}{2 i}\left(D_{e f}^{+}-D_{e f}^{-}\right) .
$$

During a time interval $t$ the phase of this probe increases by the amount

$$
\Delta \varphi=\omega_{e f} t+\frac{\Omega^{2} n}{\delta} t
$$

when the signal field contains $n$ photons. A measurement of $\Delta \varphi$ thus constitutes a measurement of $n$. This measurement obviously satisfies all the QND criteria. This results from the complete analogy with the Kerr effect discussed above, the operators $D_{e f}^{+}$and $D_{e f}^{-}$merely replacing the $a_{P}^{\dagger}$ and $a_{P}$ ones.

\section{Orders of magnitude}

Before analyzing in more detail the procedure that can be used to detect the phase shift given by Eq. (18), it is important to discuss its order of magnitude in a favorable case. Choose for the cavity a cylinder of length $L_{c}$, sustaining the $\mathrm{TE}_{121}$ mode (this is the field configuration of micromasers experiments [18]). Let us assume, for definiteness, that $e$ and $i$ are circular Rydberg levels [19] of an alkali atom, with principal quantum numbers 50 and 51 . The cavity frequency is then close to $51.1 \mathrm{GHz}$ and its length $L_{c}$ is of the order of $1 \mathrm{~cm}$. In this case [5],
$\Omega(\mathbf{r}=0)$ (Rabi frequency at cavity center) is $4.2 \times 10^{5} \mathrm{~s}^{-1}$. We obtain a shift per photon at cavity center:

$$
\Delta_{e}(n=1, \mathbf{r}=0)=\Omega^{2}(\mathbf{r}=0) / \delta,
$$

which, for $\delta=10 \Omega(\mathbf{r}=0)$, is equal to $4.2 \times 10^{4} \mathrm{~s}^{-1}$.

Assume that the atom crosses at velocity $v_{0}$ the length $L_{c}$ of the cavity mode. The accumulated phase shift per photon $\epsilon$ is then

$$
\epsilon=\overline{\Delta_{e}(n=1, \mathbf{r})} L_{c} / v_{0}=\frac{\Omega^{2}(\mathbf{r}=\mathbf{0})}{2 \delta} \frac{L_{c}}{v_{0}},
$$

where the overbar denotes a spatial average of the shift along the path of the atom. $\Omega(\mathbf{r})$ varying as a sine arch in this mode, this average amounts to dividing by 2 the shift at the cavity center. We find that the accumulated shift per photon is equal to $\pi$ for $v_{0}=70 \mathrm{~m} / \mathrm{s}$. Such a velocity can be easily obtained by laser-cooling techniques of an atomic beam [20]. Large phase shifts per photon may thus be obtained with this realistic system. Obvious$1 y$, we have considered here circular Rydberg atoms because the requirement of long radiative lifetimes is important [19]. The atoms must cross the cavity in a time of the order of $10^{-4} \mathrm{~s}$ without appreciably decaying towards more bound atomic energy levels, thus precluding the use of ordinary low-angular-momentum Rydberg atoms for this experiment.

A large $\epsilon$ value is obtained by choosing a relatively small detuning $\delta$. The detuning, however, must be a few times larger than the Rabi frequency $\Omega \sqrt{n}$ corresponding to the field we intend to measure. Smaller $\delta$ values should be avoided because they would result in an appreciable transition probability from level $e$ to $i$ during the atom-cavity coupling time, i.e., in a change of the photon number in the cavity. In other words, we have implicitly assumed in the above analysis that, as the atom crosses a cavity with $n$ photons, the atom-field coupling is switched on and off adiabatically, so that an atom introduced in level $e$ leaves the cavity in the same state, without changing the photon number. If $\delta$ becomes too small, this adiabatic condition is bound to break down and the system initially prepared in $e$ may end up in level $i$, after having absorbed one photon from the field. It is simple to compute numerically the system evolution for an arbitrary detuning $\delta$. The calculation must take into account the variation of the coupling $\Omega$ as a function of time, which reflects the fact that the atom is flying across a positiondependent cavity mode. We have performed such calculations for $\delta=3 \Omega \sqrt{n}$ and checked that the $e \rightarrow i$ transition probability remains smaller than $10^{-4}$, which validates the adiabatic approximation for this problem.

We must stress that the smooth variation of the field mode amplitude along the path of the atoms is an important feature of this experiment. For a square-shaped mode corresponding to a sudden switching on and off of the atom-field coupling, the $e \rightarrow i$ transition probability for the same $\delta$ value is $10^{-1}$, precluding the use of such modes for an efficient QND field-detection scheme. 


\section{Detection of the atomic phase by the Ramsey method}

The simplest way to detect the dephasing accumulated between the states $e$ and $f$ when the atoms cross the mode is obviously the Ramsey method of separated oscillatory fields [12]. The experimental scheme is shown in Fig. 2. Before entering the cavity, each atom is prepared by laser excitation and microwave transfers [19] into the circular Rydberg level $e$. It then interacts in a first zone $R_{1}$ in front of the cavity with an auxiliary microwave field, tuned at a frequency $\omega_{r}$, resonant or quasiresonant with the $e \rightarrow f$ transition. The atom leaves this zone in a linear superposition of levels $e$ and $f$. It then crosses the cavity and interacts downstream with a second auxiliary field, identical to the first one, in zone $R_{2}$. The distance between the two zones is $L>L_{c}$. In the absence of photons in the cavity, the phase difference between the atomic dipole and the auxiliary "Ramsey field" reference is, for an atom with velocity $v_{0}$,

$$
\varphi_{0}=\left(\omega_{r}-\omega_{e f}\right) L / v_{0} \text {. }
$$

In the presence of $n$ photons in the cavity, this phase difference becomes

$$
\varphi_{n}=\varphi_{0}-n \epsilon \text {. }
$$

To read $\varphi_{n}$ out, we analyze the state of the atom downstream, after zone $R_{2}$. If an atom with velocity $v$ crosses the cavity containing $n$ photons, the system ends up in the superposition state:

$$
\begin{aligned}
\left|\Psi_{\text {final }}^{\text {field }+ \text { atom }}\right\rangle= & b_{e}\left(n, v ; \varphi_{0}, \epsilon\right)|e, n\rangle \\
& +b_{f}\left(n, v ; \varphi_{0}, \epsilon\right)|f, n\rangle
\end{aligned}
$$

with the amplitudes $b_{e}$ and $b_{f}$ given, in interaction representation, by

$b_{e}\left(n, v ; \varphi_{0}, \epsilon\right)=e^{-i \varphi_{0} v_{0} / v}\left[\cos ^{2} \frac{\pi v_{0}}{4 v} e^{i \varphi_{n} v_{0} / v}-\sin ^{2} \frac{\pi v_{0}}{4 v}\right)$,

$b_{f}\left(n, v ; \varphi_{0}, \epsilon\right)=-\frac{1}{2} \sin \frac{\pi v_{0}}{2 v}\left(1+e^{i \varphi_{n} v_{0} / v}\right)$.

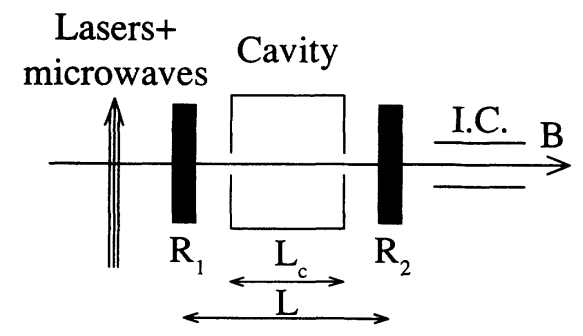

FIG. 2. Schematics of the DAP-QND experiment. The atoms of the beam $B$ are excited by lasers and microwave transfers into the circular Rydberg state $e$, cross zone $R_{1}$, the cavity, and zone $R_{2}$ before their state (e or $f$ ) and velocity $v$ are determined by the field ionization counter I.C.
These equations are derived in the Appendix, under the assumption that $\left|\omega_{r}-\omega_{e f}\right|$ is much smaller than the reciprocal of the time spent by the atom in each Ramsey zone. Similar equations have been discussed in [21]. We have assumed that an atom with velocity $v_{0}$ undergoes an exact $\pi / 2$ pulse in each Ramsey zone. The $v_{0} / v$ factors in Eqs. (24) account for the velocity dependence of the cavity crossing time. Note in Eq. (23) that the photon number is not changed by the nonresonant interaction.

The atoms are detected after zone $R_{2}$ by a fieldionization detector (I.C. in Fig. 2), which discriminates between levels $e$ and $f$. By pulsing the laser excitation and measuring the arrival time of the atoms in the detector, it is also possible to determine their velocity $v$. The probability $\Pi_{f}\left(n, v ; \varphi_{0}, \epsilon\right)\left[\Pi_{e}\left(n, v ; \varphi_{0}, \epsilon\right)\right]$ of detecting in level $f(e)$ an atom having crossed with velocity $v$ the cavity containing $n$ photons is

$$
\begin{aligned}
\Pi_{f}\left(n, v ; \varphi_{0}, \epsilon\right) & =1-\Pi_{e}\left(n, v ; \varphi_{0}, \epsilon\right)=\left|b_{f}\left(n, v ; \varphi_{0}, \epsilon\right)\right|^{2} \\
& =\sin ^{2}\left(\frac{\pi v_{0}}{2 v}\right] \cos ^{2}\left[\varphi_{n} \frac{v_{0}}{2 v}\right] .
\end{aligned}
$$

$\Pi_{f}\left(n, v ; \varphi_{0}, \epsilon\right)$ is a periodic function of $\varphi_{n}$ exhibiting a characteristic pattern of fringes whose spacing depends upon $v$. It appears equivalently as an oscillating function of $\varphi_{0}$ (i.e., of the Ramsey field frequency $\omega_{r}$ ) for a given $n$ or as an oscillating function of $n$ for a given $\varphi_{0}\left(\omega_{r}\right)$. For a field with a photon-number distribution $p(n)$, the transition probability is defined as

$$
\Pi_{f}\left(p(n), v ; \varphi_{0}, \epsilon\right)=\sum_{n} p(n) \Pi_{f}\left(n, v ; \varphi_{0}, \epsilon\right) .
$$

For a fixed $\varphi_{0}$, the above expression appears as a weighted sum over $n$ of identical fringe patterns translated by $n \epsilon$.

Next, consider the transition rate averaged over a Maxwellian atomic velocity distribution $\mathscr{D}(v)$ (thermal beam of atoms with mean velocity equal to $v_{0}$ ). For each $n$ value, we obtain a velocity-averaged probability:

$$
\Pi_{f}\left(n ; \varphi_{0}, \epsilon\right)=\int \mathscr{D}(v) \Pi_{f}\left(v, n ; \varphi_{0}, \epsilon\right) d v .
$$

The variation of $\Pi_{f}\left(n ; \varphi_{0}, \epsilon\right)$ versus $\varphi_{0}$ for $n$ fixed is represented in Fig. 3(a). Only the central fringe centered around $\varphi_{0}-n \epsilon=0$ survives the velocity averaging and the probability exhibits a single sharp feature whose position is characteristic of the photon number (the figure
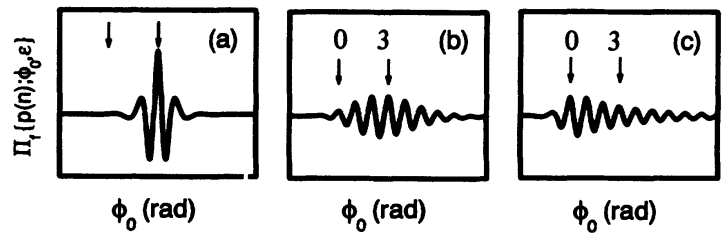

FIG. 3. Transition probability from $e$ to $f$ vs $\varphi_{0}$ for $\epsilon=2 \pi$ averaged over the atomic velocity distribution. (a) Field in a Fock state; (b) coherent field; (c) thermal field. Mean photon number in all traces: $\bar{n}=3$. Arrows indicate values $\varphi_{0}=n \epsilon$ for $n=0$ and 3. In each part, the horizontal scale is from $-6 \pi$ to $+18 \pi$ and the vertical scale is from 0 to 1 . 
corresponds to $n=3$ and the fringe position for an empty cavity is shown by an arrow; the spectrum in the figure is computed with $\epsilon=2 \pi$ ). Let us finally give the averaged probability for a thermal atomic beam interacting with a cavity field in a state described by a photon-number distribution $p(n)$ :

$$
\begin{aligned}
\Pi_{f}\left(p(n) ; \varphi_{0}, \epsilon\right) & =\sum_{n} p(n) \Pi_{f}\left(n ; \varphi_{0}, \epsilon\right) \\
& =\int d v \mathcal{D}(v) \Pi_{f}\left(p(n), v ; \varphi_{0}, \epsilon\right) .
\end{aligned}
$$

$\Pi_{f}\left(p(n) ; \varphi_{0}, \epsilon\right)$ is a superposition of single photon features, weighted by the corresponding photon-number probabilities. The variations of $\Pi_{f}\left(p(n) ; \varphi_{0}, \epsilon\right)$ versus $\varphi_{0}$ for a coherent and a thermal field [Poisson or exponential $p(n)$ distributions, respectively] are shown in Figs. 3(b) and 3(c). As noticed in [5], we see that the shape of the fringe pattern allows us to distinguish easily a coherent from a thermal or a Fock state with the same mean energy. In order to determine $\Pi_{f}\left(p(n) ; \varphi_{0}, \epsilon\right)$, we must detect a sequence of atoms for each value of $\varphi_{0}$ and determine the mean $e \rightarrow f$ transfer rate. This implies that the field is prepared in the same initial state, corresponding to $p(n)$, before each atom crosses the cavity. In a real experiment, this means that the field has the time to relax back to an equilibrium state between consecutive atoms, with negligible damping, however, during the time $L_{c} / v$ ("moderate" $Q$ cavity fed by a stationary microwave source). In practice, the recording with an acceptable signal-to-noise ratio of the curves shown in Fig. 3 would imply the detection of about $10^{5}$ atoms. Note that this experiment does not require any control of the atomic velocity. On the contrary, the dispersion of atomic velocities helps, since it makes it easy to determine the center fringe for each photon number $n$.

\section{E. Intepretation in terms of an atomic interference process}

The separated oscillatory field method just described may be conveniently described as an atomic interference process. The probability amplitude $b_{f}\left(n, v ; \varphi_{0}, \epsilon\right)$ of finding in level $f$ an atom initially prepared in $e$ is the sum of two terms which can be symbolized by the diagrams shown in Fig. 4. The atom may be transferred from $e$ to $f$ either in the first Ramsey zone [Fig. 4(a)] or in the second one [Fig. 4(b)]. In the latter case, its propagator is phase shifted by the angle $\varphi_{n} v_{0} / v$, which corresponds to the $\exp \left(i \varphi_{n} v_{0} / v\right)$ term in parentheses in Eq. (24b). In the path corresponding to Fig. 4(a), on the other hand, the cavity field has no effect on the propagator, since the atom in level $f$ is not appreciably coupled to the field [term 1 in the parentheses in Eq. (24b)]. The $\Pi_{f}\left(n, v ; \varphi_{0}, \epsilon\right)$ transition probability is the squared sum of the partial amplitudes associated to these two diagrams and the fringe pattern exhibited by Eq. (25) corresponds to their product. In other words, the atom has been prepared in zone $R_{1}$ in a coherent superposition of two states, one that "feels" the cavity field and another that does not. When the atom is finally measured in level $f$ or $e$, it is not possible to tell through which channel the sys-

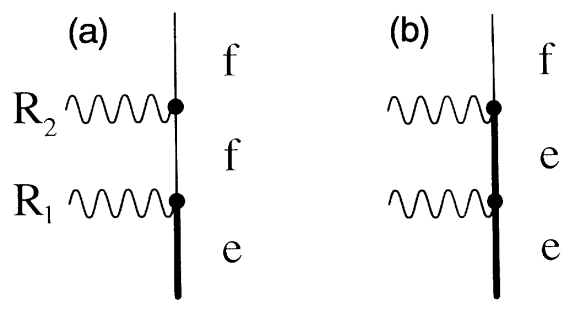

FIG. 4. Diagrams illustrating the interfering transition amplitudes in the Ramsey experiment. (a) The atom undergoes a transition in $R_{1}$ and crosses the cavity in state $f$. (b) The atom crosses the cavity in state $e$ and undergoes the $e \rightarrow f$ transition in $R_{2}$.

tem has evolved and the resulting detection probability reveals the corresponding quantum interference under the form of a Ramsey fringe pattern. We thus propose here using a matter interference effect in order to study the properties of a field, in a way quite similar to more usual interfering schemes in which light interference phenomena are used to determine the properties of a material retarding plate. There is, for example, a striking analogy with the birefringence phenomenon in optics: a linearly polarized photon whose electric field is initially at $45^{\circ}$ from the axis of a birefringent plate is the linear superposition of photons polarized along the slow and fast axis. The photon propagator through the plate is the sum of two amplitudes dephased by different amounts proportional to the plate thickness $l$. As a result, the probability of detecting the photon with a given polarization after transmission is an oscillating function of $l$ and this parameter can easily be deduced from the characteristics of this function. In our proposal, the roles of the field and matter are simply exchanged and the "length" of the retarding plate is replaced by the intensity of the cavity field. Note also that in both cases the matter-field interaction is not resonant: the usual retarding plate is a transparent medium that does not "heat." To take the analogy further, we can remark that if the plate did absorb energy, it would expand and $l$ would change, resulting in a trivial "back action" of the measuring process on the observed quantity. The analogy cannot be carried too far: in usual interferometric experiments, the measured quantity (optical length of a plate) is essentially classical, whereas in the DAP-QND scheme the observed "object" is inherently quantum: the cavity field, especially if the number of photons is small, exhibits large fluctuations and is transformed in a fundamentally random way by the measuring process. This leads to interesting consequences in experiments in which we use the atoms to monitor continuously the field, as discussed in the next section.

\section{CONTINUOUS QND MEASUREMENTS OF THE PHOTON NUMBER}

We have described an experiment performed on an ensemble of identical realizations of a field. More interestingly, the same apparatus can also be used to perform a 
quantum-mechanical measurement of the field photon number, on a single realization of this system.

\section{A. Simple analysis: Evolution of a "pure state" field}

Let us assume that the field in the cavity is described by a "pure" superposition of photon number states, which, in interaction representation, is

$$
|\Psi\rangle=\sum_{n} c_{n}|n\rangle \text {. }
$$

As an example of such a field, let us mention the coherent Glauber state [22] corresponding to a complex amplitude $\alpha$, with $c_{n}$ coefficients given by

$$
c_{n}=\frac{e^{-|\alpha|^{2} / 2} \alpha^{n}}{\sqrt{n !}}
$$

After interaction with an atom with velocity $v$, the state of the atom-field system is readily obtained by superposition of Eq. (23) type solutions:

$$
\left|\Psi_{\text {final }}^{(\text {field }+ \text { atom })}\right\rangle=\sum_{n} \sum_{\substack{a \\ a=e, f}} c_{n} b_{a}\left(v, n ; \varphi_{0}, \epsilon\right)|a, n\rangle .
$$

This is obviously an entangled state which cannot be expressed as a product of atom and field contributions: the nonresonant atom-field interaction builds strong correlations between the two parts of the coupled subsystems.

According to the postulates of a quantum measurement, the detection of the atom in level $a$ ( $a=e$ or $f$ ) projects this state into $\sum_{n} c_{n} b_{a}\left(n, v ; \varphi_{0}, \epsilon\right)|a, n\rangle$, resulting in the system "disentanglement" and the collapse of the field into a coherent superposition of Fock states with

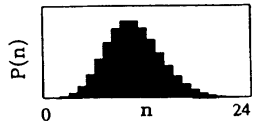

(a)

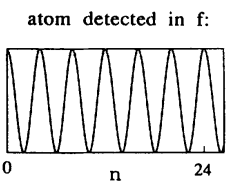

atom detected in $\mathrm{e}$ :

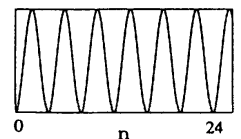

(b)
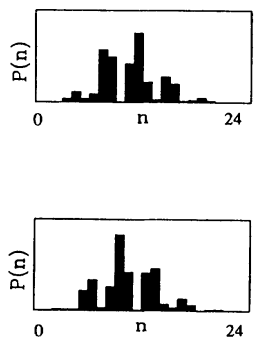

(c)
FIG. 5. Illustration of the basics of the DAP-QND method, showing the transformation of the photon-number distribution produced by a single atom detection event. The initial distribution, displayed in (a), is a Poisson law with $\bar{n}=10$. It is multiplied by the oscillating fringe function $\left|b_{a}\left(n, v ; \varphi_{0}, \epsilon\right)\right|^{2}$, represented vs $n$ in (b), for both the $f$ and $e$ detection outcomes $\left(v=v_{0}, \varphi_{0}=0, \epsilon=\pi / 2\right)$. In the resulting distributions (c), photon numbers closest to the "dark fringes" have been decimated. The patterns obtained after detection of the atom in $f$ and $e$ are "complementary." amplitudes given within a normalization factor by $c_{n} b_{a}\left(n, v ; \varphi_{0}, \epsilon\right)$. The photon-number distribution is thus essentially multiplied by an oscillating function of $n$, $\left|b_{a}\left(n, v ; \varphi_{0}, \epsilon\right)\right|^{2}$. We show in Fig. 5 how the photonnumber distribution is transformed after detection of an atom in state $f$ or $e$. The photon numbers for which the fringe function is closest to zero are efficiently decimated, since the measurement process gives us an information "incompatible" with these numbers. This decimation process is at the heart of the QND scheme studied in this article: if the process is repeated on the same field with a succession of atoms having different velocities, which are detected randomly in levels $e$ or $f$ according to the corresponding quantum-mechanical probabilities, other photon numbers are suppressed and we conceive easily that the decimation goes on until only one photon number is left. The field state is finally projected on an intensity eigenstate, even though no energy has been exchanged between the atoms and the field. The change in the field photon probability distribution has been achieved only through a dissipation free "information-gathering" process, which appears as a perfect quantum measurement of the field intensity.

This analysis, which contains the essence of our method, is only qualitative. It does not describe the measurement effects on an incoherent field, such as a thermal field, for which a "pure state" description is inadequate. Even for an initially "pure state," the above approach is incomplete because it cannot account for interactions with undetected atoms whose effect is, as discussed below, to transform the pure state into a statistical mixture. Also, it is not adapted to describe field relaxation which, in a real cavity, adds its effect to the measurement-induced processes. For a quantitative description of the method, we have to adopt a densitymatrix description.

\section{B. Evolution of the field density operator under continuous atomic detection}

Assume that a field has been prepared in a state described by a density matrix $\rho_{0}$ inside a cavity whose $Q$ factor is first supposed to be infinite. A sequence of atoms is sent through the cavity. The velocity and the quantum state of each atom are determined by the atomic detector I.C. To account for finite detection efficiency, we also allow for some "unread" events in which the atomic internal energy and velocity are not measured. As in other similar problems dealing with continuous photodetection [23,24], each atom measurement provides information on the cavity field and changes the field density operator, $\rho_{0}$ becoming $\rho_{1}$ after the first atom $\cdots \rho_{k}$ after the $k$ th atom. The state of the atom-field system at the entry of atom $k+1$ is described by the total density operator $\rho_{k}^{\text {(field +atom): }}$

$$
\rho_{k}^{(\text {field +atom })}=\sum_{n, n^{\prime}}|e, n\rangle \rho_{k ; n, n^{\prime}}\left\langle e, n^{\prime}\right|,
$$

which, after the coherent evolution of the atom-field system, becomes 


$$
\begin{aligned}
\rho_{k+1}^{(\text {field + atom })}=\sum_{n, n^{\prime}} & {\left[b_{e}\left(n, v ; \varphi_{0}, \epsilon\right)|e, n\rangle+b_{f}\left(n, v ; \varphi_{0}, \epsilon\right)|f, n\rangle\right] \rho_{k ; n, n^{\prime}} } \\
& \times\left[\left\langle e, n^{\prime}\right| b_{e}^{*}\left(n^{\prime}, v ; \varphi_{0}, \epsilon\right)+\left\langle f, n^{\prime}\right| b_{f}^{*}\left(n^{\prime}, v ; \varphi_{0}, \epsilon\right)\right],
\end{aligned}
$$

with the $b_{e}$ and $b_{f}$ amplitudes given by Eqs. (24). If the atom is detected in level $a(a=e, f)$ with velocity $v$, the total system density operator collapses into the subspace projecting on the diadics $|a, n\rangle\left\langle a, n^{\prime}\right|$ and the field density matrix become

$\rho_{k+1 ; n, n^{\prime}}^{(a, v)}=\frac{b_{a}\left(n, v ; \varphi_{0}, \epsilon\right) b_{a}^{*}\left(n^{\prime}, v ; \varphi_{0}, \epsilon\right)}{\sum_{n}\left[\left.b_{a}\left(n, v ; \varphi_{0}, \epsilon\right)\right|^{2} \rho_{k ; n, n}\right.} \rho_{k ; n, n^{\prime}}$.

The denominator in this expression ensures the normalization of the trace of the corresponding matrix. It also has a very simple physical interpretation. It is precisely the probability $\Pi_{a}\left(p_{k}(n), v ; \varphi_{0}, \epsilon\right)$ that the atom with velocity $v$ is found in state $a$ after it interacts with a field having a photon-number distribution $p_{k}(n)=\rho_{k ; n, n}$. We thus find the remarkably simple result

$\rho_{k+1 ; n, n^{\prime}}^{(a, v)}=\frac{b_{a}\left(n, v ; \varphi_{0}, \epsilon\right) b_{a}^{*}\left(n^{\prime}, v ; \varphi_{0}, \epsilon\right)}{\Pi_{a}\left(p_{k}(n), v ; \varphi_{0}, \epsilon\right)} \rho_{k ; n, n^{\prime}}$.

If only the atomic velocity is measured but not the atomic energy, the field density operator is the weighted average of the results corresponding to the $e$ and $f$ outcomes:

$$
\begin{aligned}
& \rho_{k+1 ; n, n^{\prime}}^{(a ?, v)}=\left(\sum_{a=e, f} b_{a}\left(n, v ; \varphi_{0}, \epsilon\right)\right. \\
&\left.\times b_{a}^{*}\left(n^{\prime}, v ; \varphi_{0}, \epsilon\right)\right] \rho_{k ; n, n^{\prime}} .
\end{aligned}
$$

Finally, if the atomic velocity also remains undetected, the field density operator becomes

$$
\begin{aligned}
\rho_{k+1 ; n, n^{\prime}}^{(a ?, v ?)}=\int d v \mathcal{D}(v)\left(\sum_{a=e, f} b_{a}\left(n, v ; \varphi_{0}, \epsilon\right)\right. \\
\left.\quad \times b_{a}^{*}\left(n^{\prime}, v ; \varphi_{0}, \epsilon\right)\right] \rho_{k ; n, n^{\prime}} .
\end{aligned}
$$

\section{Field collapse into a Fock state}

Equations (35)-(37) summarize the effect on the field density operator of any possible atomic event. If we are interested only in the field photon-number distribution $p(n)=\rho_{n, n}$, we merely have to particularize these equations for the field density-matrix diagonal elements. Combining Eqs. (35) with (25), we find that $p_{k}(n)$ is multiplied by $\Pi_{a}\left(n, v ; \varphi_{0}, \epsilon\right) / \Pi_{a}\left(p_{k}(n), v ; \varphi_{0}, \epsilon\right)$ if the atom is detected in level $a$ with velocity $v$. If the atomic energy is not measured, Eq. (36) combined with the identity $\Pi_{e}\left(n, v ; \varphi_{0}, \epsilon\right)+\Pi_{f}\left(n, v ; \varphi_{0}, \epsilon\right)=1$ shows that $p_{k}(n)$ is not altered at all, whether the atomic velocity is measured or not. These results are summarized by the following equations:

$$
\begin{aligned}
& p_{k+1}^{(a, v)}(n)=\frac{\Pi_{a}\left(n, v ; \varphi_{0}, \epsilon\right)}{\Pi_{a}\left(p_{k}(n), v ; \varphi_{0}, \epsilon\right)} p_{k}(n), \\
& p_{k+1}^{(a q, v ?)}(n)=p_{k+1}^{(a ?, v)}(n)=p_{k}(n) .
\end{aligned}
$$

Note that, even if no photon is exchanged between the field and the "measuring" atoms, the mere fact that information is acquired on the field has, in general, the effect of modifying its photon distribution. However, no change in this distribution occurs if the field is in a welldefined Fock state, i.e., if $p_{k}(n)=\delta\left(n-n_{k}\right)$. Equation (26) then shows that $\Pi_{a}\left(p_{k}(n), v ; \varphi_{0}, \epsilon\right)=\Pi_{a}\left(n_{k}, v ; \varphi_{0}, \epsilon\right)$ and Eq. (38) entails that a measurement of the atom in level $a$ does not modify $p_{k}(n)$ in this case. This is typical of a QND process: if the system is in a Fock state, this state must, in the absence of relaxation, remain stable forever. The fact that an unread atom does not change the photon distribution [Eq. (39)] is also characteristic of the QND nature of the process. It is instructive to compare these results with those obtained in papers discussing the continuous monitoring of a micromaser [23] or, more generally, models describing the continuous measurement of photons by a sequence of photodetection processes [24]. In these cases, Fock states are generally altered by an atomic detection event. Furthermore, even an unread atom modifies in general the field energy in the cavity and thus changes the photon-number distribution.

The above discussion leads directly to a simple recipe [5] to simulate with a computer a continuous QND measurement of the field, initially in a state corresponding to the photon-number distribution $p_{0}(n)$. We first draw a random velocity $v_{1}$ and compute $\Pi_{f}\left(p_{0}(n), v_{1} ; \varphi_{0}, \epsilon\right)$ from Eq. (26). We then decide the outcome $a$ of the first $f / e$ measurement by comparing this probability to a random number between 0 and 1 . We next multiply $p_{0}(n)$ by $\Pi_{a}\left(n, v_{1} ; \varphi_{0}, \epsilon\right)$ and we normalize by dividing by $\Pi_{a}\left(p_{0}(n), v_{1} ; \varphi_{0}, \epsilon\right)$. This yields $p_{1}(n)$. We then draw a second velocity $v_{2}$, compute $\Pi_{f}\left(p_{1}(n), v_{2} ; \varphi_{0}, \epsilon\right)$ from Eq. (26), and so on. In this way we generate $p_{2}(n) \cdots p_{k}(n)$. The computer generates for each sequence of $k$ detected atoms a set of $\left\{a_{p}, v_{p}\right\}$ values $(1<p<k)$. We call this set a " $k$-atom measuring sequence." It is easy to show by iteration that such a measuring sequence corresponds to the final photon-number distribution:

$$
p_{k}(n)=\frac{\mathscr{F}\left(n ;\left\{a_{p}, v_{p}\right\}\right) p_{0}(n)}{\sum_{n^{\prime}} p_{0}\left(n^{\prime}\right) \mathcal{F}\left(n^{\prime} ;\left\{a_{p}, v_{p}\right\}\right)}
$$

with

$$
\mathcal{F}\left(n ;\left\{a_{p}, v_{p}\right\}\right)=\prod_{p=1, k} \Pi_{a_{p}}\left(n, v_{p} ; \varphi_{0}, \epsilon\right) .
$$

This result has been obtained in [5] by a simple probabilistic argument.

Simulations of such continuous field measurements 
have been carried out for various $\varphi_{0}$ and $\epsilon$ values. The initial field can be either coherent or thermal, or described by a flat distribution of $n$ values, ranging from zero to a few tens. Quite generally, $p_{k}(n)$ is found to converge towards a distribution representing a Fock state somewhere within the width of the initial $p_{0}(n)$ distribution. Figure 6 shows an example of such a measuring sequence. The initial photon-number distribution corresponds to a coherent state with $\bar{n}=5$ [Fig. 6(a)]. Figures $6(\mathrm{~b})-6(f)$ show the distribution $p_{k}(n)$ for $k=1,3,6,10$, and 15. The field ends up in this case into the $n=3$ Fock state. Another simulation converges into another Fock state, after a number of atoms of the same order. The result of a given measuring sequence is unpredictable, and we have checked that the statistics of an ensemble of such simulations carried out on the same initial field reproduces the photon distribution $p_{0}(n)$.

The mechanism of the gradual field collapse into a Fock state has already been briefly discussed for a simple "pure state field" in Sec. IV A. The process is the same for a field described by a density matrix. Let us analyze it in more detail here. Each atomic detection results in multiplying $p(n)$ by a function of $n$ which exhibits a fringelike structure, with periodic minima whose positions change with the atomic velocity. As successive atoms with random velocities pass through the cavity and are detected, the iterative product $\mathscr{F}\left(n ;\left\{a_{p}, v_{p}\right\}\right)$ evolves after a few steps into a complicated oscillating function of $n$. As more atoms are detected, only isolated peaks survive in $\mathcal{F}\left(n ;\left\{a_{p}, v_{p}\right\}\right)$, with an average spacing between peaks $\delta n\left(\left\{a_{p}, v_{p}\right\}\right)$. This spacing increases rapidly with

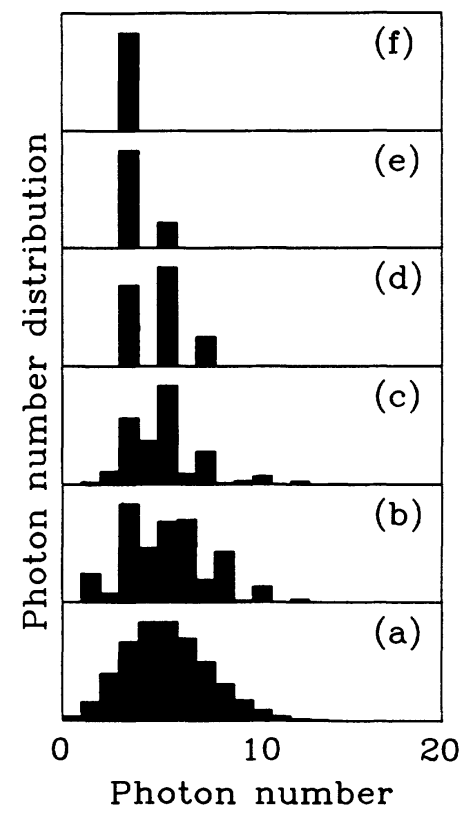

FIG. 6. Evolution of the photon-number distribution in a QND sequence. The initial state is coherent with $\bar{n}=5$ (a). Traces $(b)-(f)$ correspond to the detection of $1,3,6,10$, and 15 atoms, respectively. In this realization, the field finally collapses in the $n=3$ Fock state. the number of atoms $k$ in the sequence. The reduction into a Fock state is effective when $\delta n\left(\left\{a_{p}, v_{p}\right\}\right)$ exceeds $\Delta(n)$, the photon-number dispersion in the initial field. Practically, with fields having an average photon-number fluctuation of the order of 5 , this requires atomic samples with $k$ of the order of 10 to 15 (see Fig. 6). Let us note again that as soon as only one photon number is left, this number remains stable thereafter, at least in the absence of any other cause of evolution such as relaxation (see Sec. IV D). Note also that "unread" atoms, which do not modify $p(n)$, have no effect on this process.

Statistics performed on a large set of simulations show that the average "size" $\bar{k}$ of the measuring sequence required to reduce the field to a Fock state increases logarithmically with the photon-number dispersion in the initial field. We have assumed a total indeterminacy in the initial photon number, from 0 to a value $n_{\max }$. The simulations have been performed for $\epsilon=\pi, \varphi_{0}=0$, and a thermal velocity distribution. Figure 7 represents the average number of atoms required to produce a Fock state with a $99 \%$ probability, as a function of $n_{\max }$. Consistently with Fig. $6, k$ is of the order of 20 for $n_{\max }=10$. About 50 atoms are required, on the average, to "pin down" a field with a $\Delta(n) \simeq 500$ photon-number dispersion.

We have also studied the variations of the average "size" $\bar{k}$ of the measuring sequence as a function of $\epsilon$, the results being shown in Fig. 8 . The simulations have been performed with a flat photon-number distribution, from 0 to $n_{\max }=10$. Not surprisingly, $\bar{k}$ diverges for $\epsilon \rightarrow 0$. More interestingly, $\bar{k}$ becomes practically constant when $\epsilon>0.2 \pi$, i.e., as soon as $n_{\max } \epsilon$ is of the order of $2 \pi$. In other words, the method converges optimally as soon as the Ramsey fringe function varies from 0 to 1 within the width of the initial photon-number distribution. The fringe signal then provides the best possible discrimination between different $n$ values.

We have considered so far the case where the atoms have random velocities, with a dispersion described by the thermal Maxwellian distribution $\mathscr{D}(v)$. Let us stress

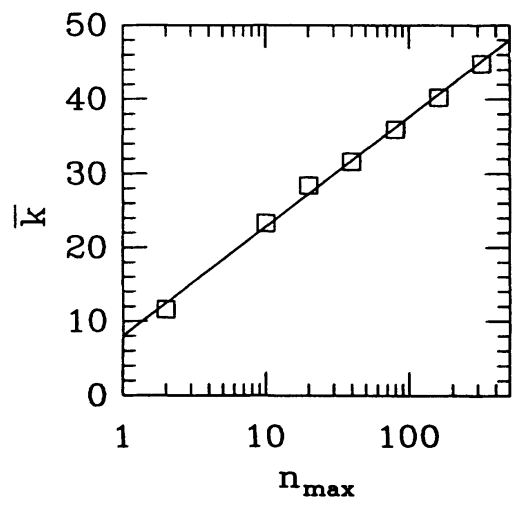

FIG. 7. Average number $\bar{k}$ of atoms in a measuring sequence, vs the initial photon number dispersion $n_{\max }$, in a semilog plot. The simulations are performed with $\epsilon=\pi$ and a thermal velocity distribution. Each box is the average of 100 simulations and the line is a best fit. 


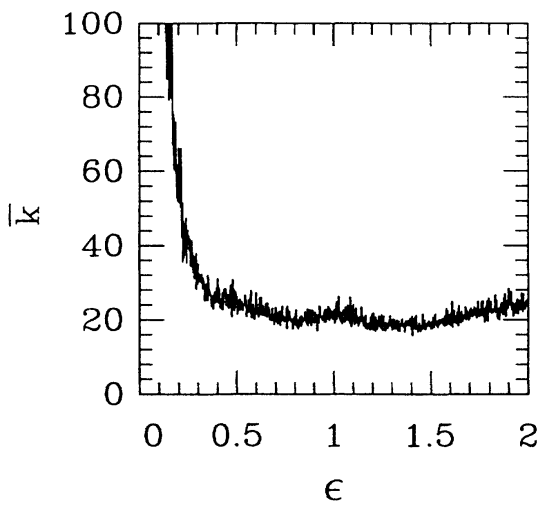

FIG. 8. Average number $\bar{k}$ of atoms in a measuring sequence, vs $\epsilon$, for $n_{\max }=10$. A thermal velocity distribution is assumed.

again the fact that this dispersion is an asset for the method, which ensures a relatively fast decimation of different photon numbers. Similar simulations show that, if the atomic beam is monokinetic, the field collapse may require a much larger atom number, or even never happen if $\epsilon$ is a rational multiple of $2 \pi$. This point will be discussed below, when we describe the field phase evolution (Sec. V).

Finally, a faster convergence of the $\mathscr{F}$ function towards a $\delta$-like distribution of $n$ may be obtained if the atomic velocity can be actively controlled and adjusted to provide for an efficient decimation of preset $n$ values at each step of the process. The procedure may also be modified by changing between atoms the Ramsey fields frequency (i.e., $\varphi_{0}$ ) or even the phase shift per photon $\epsilon$, which can be controlled by adjusting the detuning $\delta$ between the $e \rightarrow i$ atomic transition and the cavity field. We will not consider in detail here the various strategies which can be designed in order to converge as fast as possible towards a Fock state. Let us only mention that the minimum number of atoms required to ensure the field collapse cannot be smaller than $\log _{2}[\Delta(n)]$. This result has a simple interpretation in terms of "information theory." Since each atom yields a binary information (atom found in $e$ or $f$ ), the number $k$ of atoms necessary to "pin down" the field should at least be such that $2^{k}=\Delta(n)$.

In a real field measurement, the $\left\{a_{p}, v_{p}\right\}$ sequence would be measured, but $p_{0}(n)$ would be by definition unknown. We will assume, however, that we could safely place an upper limit $n_{\max }$ to the cavity field photon number (this requirement being not very restrictive since it is realistic to assume that we could have a rough idea of the field energy). According to the above analysis, we know that, after a number of atoms depending logarithmically on $n_{\text {max }}, \mathcal{F}\left(n ;\left\{a_{p}, v_{p}\right\}\right)$ would converge in the $\left[0, n_{\text {max }}\right]$ interval towards a $\delta$-like function, whose position depends only upon the $\left\{a_{p}, v_{p}\right\}$ sequence. We thus conclude that a computer interfaced to the atomic detector, which can compute the $\mathcal{F}$ function in real time, would provide an unambiguous photon-number value in the $\left[0, n_{\max }\right]$ interval, even though $p_{0}(n)$ is uncertain, which is a basic requirement for a true measurement. Of course, the "a priori probability" that a given sequence will be ob- tained depends on $p_{0}(n)$. Actually, this experiment would allow us also to determine this distribution, provided we could reproduce the initial state at will and perform a photon-number determination on each field realization.

\section{Monitoring Fock state evolution under weak relaxation}

We have assumed so far that the relaxation of the field is negligible (infinite $Q$ cavity). The above calculations can be generalized in a straightforward way to a cavity with a finite field damping time $t_{\text {cav }}$ coupled to a thermal reservoir at temperature $T$. The field density-matrix relaxation is described by a temperature-dependent Liouville operator $\Lambda_{F}$ defined as $[14,15]$

$$
\begin{aligned}
\frac{d \rho}{d t}=-\Lambda_{F} \rho & =-\frac{\kappa}{2}\left(n_{b}+1\right)\left[a_{S}^{\dagger} a_{S}, \rho\right]_{+}+\kappa\left(n_{b}+1\right) a_{S} \rho a_{S}^{\dagger} \\
& -\frac{\kappa}{2} n_{b}\left[a_{S} a_{S}^{\dagger}, \rho\right]_{+}+\kappa n_{b} a_{S}^{\dagger} \rho a_{S},
\end{aligned}
$$

where $\kappa=\omega / Q=1 / t_{\text {cav }}$ is the cavity-damping rate and $n_{b}$ is the average number of thermal photons in the mode at temperature $T$. The irreversible evolution of $\rho$ during a time interval $\delta t$ between two consecutive atoms is

$$
\rho(t+\delta t)=e^{-\Lambda_{F} \delta t} \rho(t) \simeq\left(1-\Lambda_{F} \delta t\right) \rho(t) .
$$

In order to take relaxation into account in the simulation of a continuous measurement, the density operator $\rho_{k}$ obtained after detection of atom $k$ is merely replaced by $e^{-\Lambda_{F} \delta t} \rho_{k}$ before applying the reduction procedure corresponding to the detection of atom $k+1$. This calculation describes situations where the time intervals when the cavity is empty are much longer than the times when it contains an atom. If it is not the case, one should in principle describe the combined effects of relaxation and coherent atom-field coupling during the time an atom interacts with the mode. This would considerably complicate the analysis leading to Eqs. (35) and (38).

The procedure we have outlined above avoids this difficulty by assuming a fictitious relaxation process affecting the field only between atoms and occurring with a rate $\kappa^{\prime}=\kappa / \eta$, where $\eta$ is the fraction of time during which the cavity is empty. Clearly this fictitious relaxation process is equivalent to the real one as long as Eq. (43) can be linearized, i.e., as long as the time between atoms is very short compared to $t_{\text {cav }}$. In the presence of relaxation, the reduction of the field into a Fock state will be observable provided a measuring sequence can be performed in a time short compared to the Fock state "lifetime," which is known to be of the order of $t_{\text {cav }} / n$ $[25,26]$.

Since $\epsilon=0.2 \pi$ can be achieved with $v_{0}=350 \mathrm{~m} / \mathrm{s}$ corresponding to an atom-cavity interaction time $L_{c} /$ $v_{0}=3 \times 10^{-5} \mathrm{~s}$, a measuring sequence of 20 detected atoms takes about $10^{-3} \mathrm{~s}$. The state reduction for fields with $n$ around 10 should thus be observable with cavities having a damping time $t_{\mathrm{cav}} \geq 10^{-2}$ s, i.e., a $Q$ factor at 50 $\mathrm{GHz}$ larger than $3 \times 10^{9}$. In fact, $Q=3 \times 10^{10}$ has been achieved at $21 \mathrm{GHz}$ in a superconducting cavity cooled 


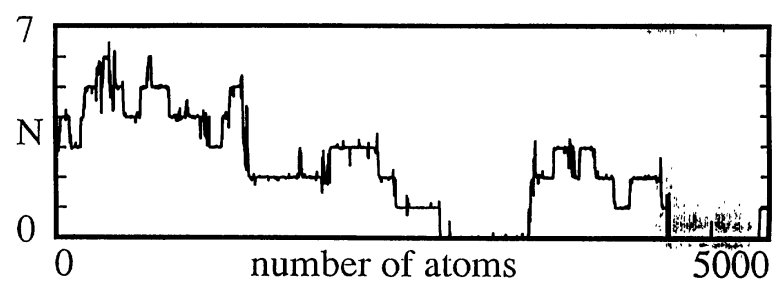

FIG. 9. Photon-number evolution in a simulation of a continuously monitored thermal field coupled into a slowly relaxing cavity $\left(\bar{n}=3, \varphi_{0}=0.15 \pi, \epsilon=\pi\right)$. The "measured" photon number (vertical axis) is plotted against the number of detected atoms. The total time scale corresponds to the passage of 5000 atoms and the relaxation time to 2500 .

at $0.5 \mathrm{~K}$ [27] and $Q$ factors of the order of $10^{10}$ are realistic at $50 \mathrm{GHz}$. The QND method using circular Rydberg atoms has thus the potential time resolution required to detect the evolution of small photon number fields.

Figure 9 represents the result of a simulation of a continuously monitored thermal field coupled into a slowly relaxing cavity. The number of photons is represented as a function of the number of detected atoms. The cavity relaxation time corresponds to the passage of 2500 atoms. The average photon number is $\bar{n}=3$, but the actually measured number fluctuates from zero to seven, the field spending in each state a time proportional to its stationary probability. Quantum jumps are visible as the photon number suddenly switches from one value to the next.

Figure 10 shows the decay of a continuously monitored field in a cavity at $T=0 \mathrm{~K}$. The field is initially in the $n=8$ Fock state. The horizontal time scale corresponds this time to the detection of 2000 atoms and the cavitydamping time to 1000 . The field intensity exhibits jumps at random times between decreasing photon numbers, until it eventually reaches zero. The usual exponential decay is recovered by ensemble averages of such realizations.

\section{BACK ACTION OF THE DAP-QND MEASUREMENT ON THE FIELD PHASE}

As atoms crossing the cavity are detected one after another and the photon number in the cavity gets re-

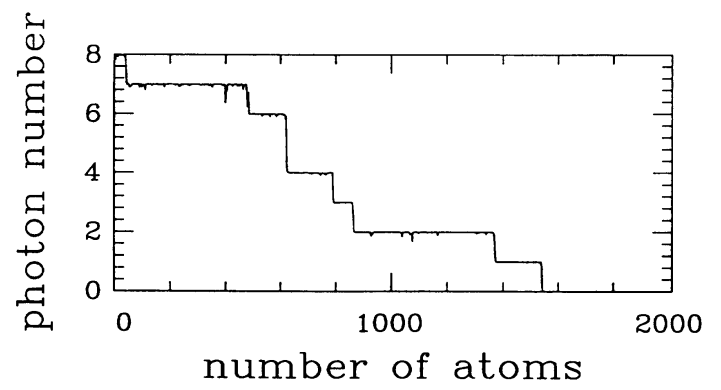

FIG. 10. Photon-number evolution in a simulation of a continuously monitored relaxing field at $T=0 \mathrm{~K}$. The initial field is the $n=8$ Fock state. The horizontal scale is the number of detected atoms. Cavity relaxation time corresponds to 1000 atoms $\left(\varphi_{0}=0.15 \pi, \epsilon=\pi\right)$. duced to a single value, we expect the phase of the field to be strongly perturbed, until it gets completely randomized in the final Fock state. In other words, the back action of the measuring process affects the field phase, which is the conjugate observable of the photon number. Moreover, even the interactions with "unread" atoms, which have no effect on the photon number distribution, do perturb the field phase. The phase diffusion can be studied by the same kind of numerical simulation as done for the photon distribution. We must now make use of the general recursion relations (35)-(37) with $b_{e}$ and $b_{f}$ given by Eqs. (24) and study the time evolution of offdiagonal matrix elements of the field density operator $\left(n^{\prime}=n+1\right.$ yields the evolution of the field amplitude). The numerical procedure is straightforward, but it is also useful to get some physical insight by discussing first simple situations lending themselves to analytical considerations.

\section{A. Evolution of $\rho_{n, n}$ : Analytical considerations}

Using Eqs. (24) and (34), the following recursion relations are obtained for $\rho_{n, n^{\prime}}$ when $v=v_{0}$ (atom undergoing a $\pi / 2$ pulse in each Ramsey zone):

$$
\begin{aligned}
& \rho_{k+1 ; n, n^{\prime}}^{\left( \pm, v_{0}\right)}=\frac{\left(1 \pm e^{i \varphi_{n}}\right)\left(1 \pm e^{-i \varphi_{n}^{\prime}}\right)}{\sum_{n} \rho_{k ; n, n}\left|1 \pm e^{i \varphi_{n}}\right|^{2}} \rho_{k ; n, n^{\prime}}, \\
& \rho_{k+1 ; n, n^{\prime}}^{\left(a ?, v_{0}\right)}=\frac{1}{2}\left(1+e^{i\left(\varphi_{n}-\varphi_{n^{\prime}}\right)}\right) \rho_{k ; n, n^{\prime}} .
\end{aligned}
$$

In Eq. (44), the + and - signs correspond, respectively, to the detection of the atom $k+1$ in level $f$ or $e$. These equations have been derived in a slightly different form in [21]. We immediately see that detecting an atom in level $e$ or $f$, as well as not detecting the atom at all, generally results in an alteration of the signal-field coherence. The evolution of the field is quite different, however, depending on whether the atomic state is measured or not. Thus, if the field is initially in a pure state, and all successive atoms are detected in either the state $|e\rangle$ or the state $|f\rangle$, the field remains in a pure state, obtained after each measurement by applying the corresponding projection operator $(|e\rangle\langle e|$ or $|f\rangle\langle f|)$ to the state just before detection. This can also be seen directly from Eq. (44): if $\rho_{k ; n, n^{\prime}}$ is factorizable as a function of $n$ times a function of $n^{\prime}$, the same is true for $\rho_{k+1 ; n, n^{\prime}}^{\left( \pm, v_{0}\right)}$ On the other hand, if the state of the atom is not determined, then the field evolves, in general, into a statistical mixture. Indeed, so long as $\epsilon$ is not a multiple of $\pi$, we have

$$
\begin{aligned}
\operatorname{Tr} \rho_{k+1}^{2} & =\sum_{n, n^{\prime}}\left|\rho_{k+1 ; n, n^{\prime}}^{\left(a ?, v_{0}\right)}\right|^{2} \\
& =\sum_{n, n^{\prime}} \cos ^{2}\left[\epsilon\left(n-n^{\prime}\right)\right]\left|\rho_{k ; n, n^{\prime}}\right|^{2}<\operatorname{Tr} \rho_{k}^{2},
\end{aligned}
$$

an inequality that is the signature of the statistical nature of $\rho_{k+1}$. The following inequality is also satisfied:

$$
\left|\rho_{k+1 ; n, n+1}^{\left(a 2, v_{0}\right)}\right|<\left|\rho_{k ; n, n+1}\right|,
$$

which means that the magnitude of the average electric 
field is reduced by an "unread" atomic event.

Equation (47) can be interpreted as resulting from a phase diffusion process, as can be shown by the following argument. Let us first make the important remark that in the case of an "unread" atom, the second Ramsey zone is obviously irrelevant. In this zone, the atoms have already interacted with the signal field and, if the information resulting from their evolution after they have left the cavity is not collected, it cannot change the knowledge we have of the field [it is indeed easy to check that Eq. (45) is not modified if just the first Ramsey zone is present]. We will thus discuss the case of "unread" atoms as if the second zone was missing. Let us assume that the initial field is in the state $\Sigma_{n} c_{n}|n\rangle$. Immediately after interacting with an "unread" atom, the field is left in the statistical mixture of the states corresponding to the two possible atomic final states. The state correlated to an atom in level $e$ is (except for a global phase)

$$
\left|\Psi_{\epsilon}^{(e)}\left(n o R_{2}\right)\right\rangle=\sum_{n} c_{n} e^{-i n \epsilon}|n\rangle .
$$

This equation shows that, if the atom were detected in level $e$, the phase of the field would be shifted by $\epsilon$. This phase shift can be interpreted as being due to a timevarying index of refraction created in the cavity by an atom in level $e$. On the other hand, the field would not be dephased at all if the atom were detected in $f$ so that $\left|\Psi_{\epsilon}^{(f)}\left(n o R_{2}\right)\right\rangle=\Sigma_{n} c_{n}|n\rangle$. Since the atom is "unread," the field turns into a statistical mixture of the unperturbed and dephased fields, whose phase becomes uncertain. Obviously, the cumulative effect of such "unread" atoms amounts to a statistical diffusion of the signal-field phase, so that the field amplitude decreases with time, as expressed by Eq. (47).

A quite different scenario emerges if the final atomic state is detected. In order to better grasp the physical difference between the two situations, let us again consider first the simpler arrangement where the second Ramsey zone is missing and the atoms detected right after they leave the cavity. Immediately after an $f$ or $e$ state detection, the field experiences an $\epsilon$ or a zero phase shift, but it remains always in a pure state $\left[\left|\Psi_{\epsilon}^{(f)}\left(n o R_{2}\right)\right\rangle\right.$ or $\left.\left|\Psi_{\epsilon}^{(e)}\left(n o R_{2}\right)\right\rangle\right]$. Contrary to what happens when the atoms are not monitored, neither the field amplitude nor the phase uncertainty are changed in this process, which may be visualized as a stepwise rotation of the field vector, which, for a monokinetic atomic beam, always has the same value, even though it occurs at random times. Of course, the average over many realizations of this experiment will reproduce the results of the "unread" case. For each realization, the field will have at the same instant of time a different phase, in such a way that the ensemble average does go to zero as $t \rightarrow \infty$.

The situation is similar, although a little bit more complicated, if the atoms are detected after the second Ramsey zone. The field being initially in a linear superposition $\sum_{n} c_{n}|n\rangle$, the state of the field after the measurement is obtained by projecting the right-hand side of Eq. (31) onto the corresponding eigenstates and normalization. Omitting a global phase factor, we get, in interac- tion picture,

$$
\left|\Psi_{\epsilon}^{( \pm)}\right\rangle=\sum_{n}\left(\frac{c_{n}\left(1 \pm e^{i\left(\varphi_{0}-n \epsilon\right)}\right)}{\left.\left.\left|\sum_{n}^{\prime}\right| c_{n}^{\prime}\left(1 \pm e^{i\left(\varphi_{0}-n^{\prime} \epsilon\right)}\right)\right|^{2}\right|^{1 / 2}}\right)|n\rangle .
$$

The + and - signs correspond, respectively, to a detection in $f$ or $e$. We see that, in both cases, the field turns into a superposition of the initial state and the state which is phase shifted with respect to it by $\epsilon$. If the initial field has a well-defined phase, such a superposition is known as a "Schrödinger cat state." Measurement of the next atom will further "split" each component of this "cat" into two subcomponents, leading to a "secondgeneration Schrödinger cat state" with four components, one of which is dephased by $2 \epsilon$ with respect to the original field, and so on. In general, this process will go on, until field components whose phases span the whole interval from 0 to $2 \pi$ are generated. This corresponds to a complete loss of phase information, an obvious consequence of the field reduction into a Fock state.

An interesting special case occurs when $\epsilon$ is a rational multiple of $\pi$. Obviously, only a finite number of different phase components are then generated by the measurement process. The reduction into a completely phase-indetermined Fock state cannot occur: the "size" of a measuring sequence with velocity-selected atoms corresponding to these special $\epsilon$ values diverges, a result already briefly mentioned in Sec. IV C.

The evolution of the field is quite different from the one in the case where the atoms are not read. In the absence of dissipation, the field indeed remains in a pure state and, although the phase uncertainty also increases (due to the appearance of multiple phase components), there is no loss of coherence between the remaining Fock states (that is, the states which have not yet been decimated as the successive atoms are measured). Note also that the phase evolution is different from the one corresponding to the case where the second Ramsey zone is missing: instead of randomly jumping from a single value to another one, the phase evolves now according to a dichotomic process.

If the atomic beam has velocity dispersion, the above discussion easily generalizes with the only difference being that the phase increments $\left(\epsilon v_{0} / v\right)$ become also random quantities. The field ends up with zero coherence whether the atoms are "read out" or not: if they are not detected, phase diffusion occurs, and if they are continuously monitored a Fock state eventually results from the generation of "multiple-component Schrödinger cats." In a realistic situation, some atoms are read and others are "missed." The phase diffusion then results from a combination of the two effects discussed above. Note that the singularities in the measuring sequence "size" do not occur in this case (see Fig. 8).

This analysis has shown that the DAP-QND process applied to an initially "pure state" field of the form (29) leads, at its successive stages, to the preparation of multiple-phase "Schrödinger cat states." These states, which have very intriguing nonclassical properties, will be studied in detail in Secs. VI and VII. 


\section{B. Numerical simulations of the Pegg-Barnett phase evolution}

In order to describe more precisely the evolution of the field, we have to resort to numerical simulations. Since it is not practical to plot all the matrix elements of $\rho$, we compute instead a combination of off-diagonal densitymatrix elements representing the field phase distribution $\Pi(\theta)$. We adopt here the formalism of the phase operator recently analyzed by Pegg and Barnett [28] and we introduce $\Pi(\theta)$ as [29]

$$
\Pi(\theta)=\lim _{s \rightarrow \infty} \frac{1}{2 \pi} \sum_{n, n^{\prime}=0}^{s} \rho_{n, n^{\prime}} e^{-i\left(n-n^{\prime}\right) \theta} .
$$

This distribution appears as a kind of Fourier transform of the off-diagonal density-matrix elements considered as a function of $n-n^{\prime}$. Without entering into all the subtleties of the Pegg-Barnett theory, let us only remark that $\Pi(\theta)$ has all the features required for a wellbehaved phase distribution. We notice, for example, from Eq. (50) that if the field is incoherent, i.e., if $\rho=\Sigma_{n} p(n)|n\rangle\langle n|$, then $\Pi(\theta)=1 / 2 \pi$, as should be expected. On the other hand, if the field is in a coherent state $|\alpha\rangle$ with a large average photon number $|\alpha|^{2}$, it is easy to show that $\Pi(\theta)$ is a sharply peaked function centered at $\theta=\phi$, where $\phi$ is the phase of $\alpha$, again an expected result.

$\Pi(\theta)$ provides a simple visualization of the phase during the QND measurement process. We merely have to

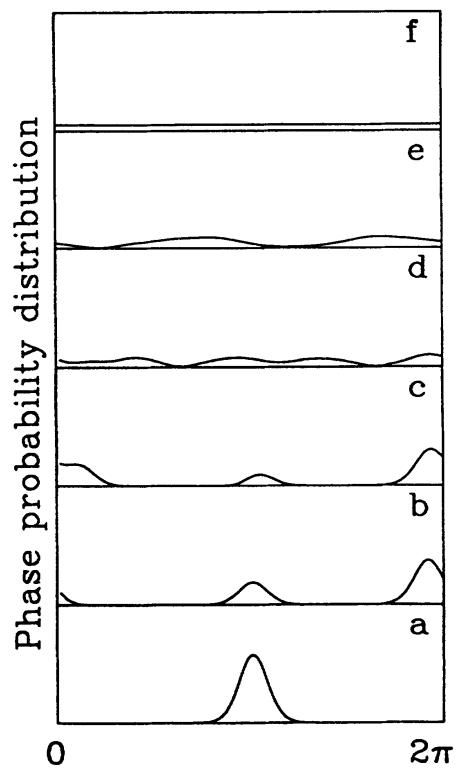

$\Theta$

FIG. 11. Evolution of the Pegg-Barnett phase distribution $\Pi(\theta)$ in the same QND sequence as in Fig. 6. All atoms are detected. $\Pi(\theta)$ for the initial coherent field is shown in (a). Traces (b)-(f) correspond, as in Fig. 6, to the detection of 1, 3, 6, 10 , and 15 atoms. The phase distribution, peaked around $\theta=0$ in trace (a), converges to a uniform distribution in trace (f). use Eqs. (35)-(37) to determine the evolution of the coherences both in the "read" and "unread" cases. The result after each step is then plugged into Eq. (50) to yield $\Pi(\theta)$. We have represented in Fig. 11 the evolution of the phase distribution for the measuring sequence whose photon-number distribution is shown in Fig. 6. Figure 11(a) shows the single-peaked $\Pi(\theta)$ distribution of the initial coherent state. Figures $11(\mathrm{~b})-11(\mathrm{f})$ show the phase distribution after $1,3,6,10$, and 15 atoms (all atoms are read in this simulation).

The detection of the first atom transforms $\Pi(\theta)$ into a double-peaked distribution characteristic of a Schrödinger cat state. Further atoms contribute to the phase scrambling and the final Fock state whose photonnumber distribution is shown in Fig. 6(f) has a completely undetermined phase, as expected [see Fig. 11(f)]. By simultaneous inspection of the plots shown in Figs. 6 and 11 we clearly observe the loss of phase information correlated to the acquisition of information on the field energy.

\section{GENERATION AND DETECTION OF SCHRÖDINGER CAT FIELD STATES}

We have shown in Sec. $V$ that a single atom interaction and detection stage of the DAP-QND scheme generally transforms a pure state field into a linear superposition of phase-shifted fields. In the remainder of this paper, we intend to study in more detail this effect, to analyze the intriguing properties of these states, and to propose experiments in which these properties could be studied.

\section{A. Interaction of a classical field with a single atom}

Let us assume that the initial field is in a coherent state $|\alpha\rangle$ with the amplitudes $c_{n}(\alpha)$ defined by Eq. (30). This field has a Poisson photon-number distribution with an average photon number $\bar{n}=|\alpha|^{2}$. It corresponds to a "classical field" which can in practice be generated by coupling an initially empty cavity with a classical current source for a time $\delta t_{\alpha}$. From Eqs. (30) and (49), we deduce that the state of the field after detection of an atom of velocity $v_{0}$ is the "Schrödinger cat state" (we assume here $\left.\varphi_{0}=0\right)$ :

$$
\left|\Psi_{\epsilon}^{( \pm)}(\alpha)\right\rangle=\frac{|\alpha\rangle \pm\left|\alpha e^{-i \epsilon}\right\rangle}{\sqrt{2\left(1 \pm \operatorname{Re}\left\langle\alpha \mid \alpha e^{-i \epsilon}\right\rangle\right)}}
$$

with the + and - signs corresponding, respectively, to a detection in level $f$ or $e$. The denominator in Eq. (51) ensures the state normalization. It depends upon the scalar product of the two phase-shifted classical field states, negligible as soon as $\bar{n}$ is large and $\epsilon$ is not too small:

$$
\operatorname{Re}\left\langle\alpha \mid \alpha e^{-i \epsilon}\right\rangle=\cos (\bar{n} \sin \epsilon) \exp [\bar{n}(\cos \epsilon-1)] .
$$

In the DAP-QND method discussed above, the atomic velocities are random and the "cat" prepared by a first atom will usually be split into more components by subsequent atomic interactions. In order to generate and conserve these states for an extended period of time, it will thus be necessary to couple the field to a single atom, or else to make use of a velocity-selected beam of atoms 
( $\left.v=v_{0}\right)$ and choose $\epsilon$ values which are rational multiples of $\pi$. In this case, the phase diffusion process analyzed in Sec. $V$ cannot randomize the field phase. The simplest situation, to which we will restrict our analysis here, is $v=v_{0}, \epsilon=\pi$. As we show below, the "first-generation cat" remains in this case stable in the absence of relaxation.

\section{B. Interaction with a "sequence" of velocity-selected atoms producing an $\epsilon=\pi$ phase shift}

Assume that the atomic velocity is set to the value $v_{0}$ and that the Ramsey fields are resonant $\left(\varphi_{0}=0\right)$, with their amplitude set so that the atoms undergo exactly a $\pi / 2$ pulse on the $e \rightarrow f$ transition in each zone. The cavity detuning $\delta$ is also set so that the atoms undergo a phase shift per photon $\epsilon$ exactly equal to $\pi$. Equations (24) then yield the following simple results for the transition amplitudes $b_{e}$ and $b_{f}$ :

$$
b_{ \pm}\left(n, v_{0} ; \varphi_{0}=0, \epsilon=\pi\right)=\mp \frac{1}{2}\left(e^{-i n \pi} \pm 1\right)
$$

(upper and lower signs correspond, respectively, to $f$ and e).

The probability of detecting the atom in $f(e)$ is thus zero for $n$ odd (even). For a field with a photon-number distribution $p_{0}(n)$, the probabilities of detecting the atom in $f$ or $e$ are, according to Eq. (26),

$$
\begin{aligned}
& \Pi_{+}\left(p_{0}(n), v_{0} ; \varphi_{0}=0, \epsilon=\pi\right)=\sum_{n \text { even }} p_{0}(n), \\
& \Pi_{-}\left(p_{0}(n), v_{0} ; \varphi_{0}=0, \epsilon=\pi\right)=\sum_{n \text { odd }} p_{0}(n) .
\end{aligned}
$$

Obviously, these probabilities are both close to 0.5 , as soon as the width of the $p_{0}(n)$ distribution is large compared to 1 . If the first atom crossing the cavity is actually measured in level $f$, the odd photon numbers are all decimated in the photon distribution, which after the measurement becomes

$$
\begin{aligned}
& p_{1}^{(+)}(n)=\frac{\left|c_{n}(\alpha)\right|^{2}}{\sum_{n \text { even }}\left|c_{n}(\alpha)\right|^{2}}(n \text { even }), \\
& p_{1}^{(+)}(n)=0 \quad(n \text { odd }) .
\end{aligned}
$$

If, on the other hand, the atom is detected in level $e$, the photon-number distribution turns into

$$
\begin{aligned}
& p_{1}^{(-)}(n)=\frac{\left|c_{n}(\alpha)\right|^{2}}{\sum_{n \text { odd }}\left|c_{n}(\alpha)\right|^{2}} \quad(n \text { odd }), \\
& p_{1}^{(-)}(n)=0 \quad(n \text { even }) .
\end{aligned}
$$

Thus, the interaction of a single atom, followed by its detection, has transformed the initial Poisson distribution into an even or an odd distribution in which only one parity of photon number is left. In fact the measuring process leaves the field in a pure state, with coherences between the remaining Fock states. This state is obtained from Eq. (51), in which we set $\epsilon=\pi$ :

$$
\left|\Psi_{\epsilon=\pi}^{( \pm)}(\alpha)\right\rangle=\frac{|\alpha\rangle \pm|-\alpha\rangle}{\sqrt{2\left(1 \pm \exp -2|\alpha|^{2}\right\rangle}}
$$

Using Eq. (30), we retrieve indeed that the photonnumber distribution is even for the + sign, odd for the sign. Furthermore, in the form of Eq. (56), the existence of coherences between the various Fock state components is clearly apparent. The special "Schrödinger cat states" obtained in this case are superpositions of classical fields with opposite phases. These are highly nonclassical fields whose properties have been studied recently in detail $[30,31]$. Let us note only here that such fields have very large fluctuations in the quadrature component, which has the phase of the initial field, and very small fluctuations in the other one.

Let us now consider the interaction of a second atom with the $\Psi_{\epsilon=\pi}^{( \pm)}(\alpha)$ cats. The probability of detecting this atom in level $f$ or $e$ is now given by Eq. (54), in which $p_{0}(n)$ is replaced by $p_{1}(n)$, given by Eq. (55a) or $(55 \mathrm{~b})$. We find the remarkable result that the probability of finding the atom in level $e$ is zero for $\Psi_{\epsilon=\pi}^{(+)}(\alpha)$, and that the probability of detecting the atom in state $f$ is zero for $\Psi_{\epsilon=\pi}^{(-)}(\alpha)$. In other words, if the first atom has been detected in level $a$ ( $e$ or $f$ ), the atom which follows will, with $100 \%$ probability be found in the same level. The photon probability distribution will thus stay unchanged in this second process. This holds of course for all subsequent atoms, provided they all produce the same $\epsilon=\pi$ phase shift per photon. In fact, the Schrödinger cat state wave function itself is unaltered by the successive atoms. Each component of the cat given by Eq. (56) is transformed by a subsequent atom in the same Schrödinger cat, so that the "first-generation cat" remains globally invariant in the process. We thus see that a beam of atoms with conveniently selected velocities produces a stable "Schrödinger cat state," whose parity is determined by the outcome of the first atomic measurement.

Note also that it is not necessary to detect the atoms with $100 \%$ efficiency to preserve the initially prepared Schrödinger cat. Since the probability of finding the atom in the other level is zero, we know for sure what the outcome of the measurement of an unread atom would have been and the system remains in the pure "Schrödinger cat state" even if the atom escapes detection (we must only be sure of its velocity). This "stability" property holds only if we can neglect cavity relaxation during the experiment. We will consider the effect of relaxation on the monitored "Schrödinger cat" in the next section.

Other mechanisms for generating "Schrödinger cats" of the electromagnetic field have been discussed in several papers over the past couple of years $[8,32,33]$. Our method has the merit of being conceptually very simple and to result in the preparation of a Schrodinger cat confined to a cavity, which could be monitored during an extended period of time. The principle of the DAP-QND scheme to prepare a stable Schrödinger cat can be summarized in the following way. We have passed through the cavity an atom in a quantum-mechanical linear superposition of two states, one of which $(e)$ dephases by $\pi$ the 
field in the cavity and the other one $(f)$ has no effect on that field. As a result of coherent mixing of the atomic states in the second Ramsey zone and detection, the quantum-mechanical superposition is no longer carried by the atom but by the field. In other words, the atomic superposition has been replicated into a field superposition. We have obtained a field which has either the initial phase, or a phase shifted by $\pi$, the either-or alternative being of a quantum-mechanical and not classical nature, since there is a nonvanishing coherence between the two states. At the same time, each of the coherent states involved in the Schrödinger cat state may be associated with a classical field, so that we are able to get in this way a quantum superposition of two classical states.

\section{Detection of the Schrödinger cat state}

How do we detect the Schrödinger cat state and demonstrate that it indeed corresponds to a coherent superposition of macroscopically different fields? The method suggested in [32] consists in measuring two quadratures of the field, of which one displays the existence of two "classical" field states and the other signals the existence of a quantum coherence between them. Field quadratures could be measured by homodyning the field under study with a reference field having either the phase of the initial coherent state (in-phase quadrature) or a phase shifted by $\pi / 2$ (out-of-phase quadrature). In the cavity QED experiments discussed in this paper, a difficulty arises from the fact that we do not have a direct access to the fields: they are "trapped" in the high- $Q$ cavity and we can only infer their properties from the detection of atoms coupled to the field during the cavity crossing time. Furthermore, only one mode of the field is available in the cavity at frequency $\omega$, which precludes realization of the usual homodyne mixing scheme. The method, however, can be adapted in the following way. After the Schrödinger cat state has been prepared, the cavity is coupled during a short time interval $\delta t_{\alpha}^{\prime}$ to the same classical source as the one which has initially been used to inject the field $|\alpha\rangle$, either directly or through a $\pi / 2$ phase shifter. A coherent field $\left|\alpha_{r}\right\rangle$ (the "reference") is thus "added" to the "cat state" in the cavity mode. The amplitude of this reference can be adjusted by varying $\delta t_{\alpha}^{\prime}$. The resulting field in the cavity, immediately after this process, is

$$
\left|\Psi^{( \pm)}\left(\alpha, \alpha_{r}\right)\right\rangle=\frac{\left|\alpha+\alpha_{r}\right\rangle \pm\left|-\alpha+\alpha_{r}\right\rangle}{\sqrt{\mathcal{N}}},
$$

where $\mathcal{N}$ is a normalization constant. Note that the addition process we are considering is quite different from the combination of fields produced by a beam splitter, which mixes together distinct modes coupled to its two "ports" and introduces vacuum noise even in the absence of any classical input field. We are describing here a much simpler field amplitude superposition mechanism, which affects only one mode of the field, coupled in succession to two classical sources through a direct waveguide-type connection, without any beamsplitter. The coupling is implicitly assumed to be very weak, so as not to limit the cavity $Q$ factor. This superposition mechanism was in fact discussed by Glauber in his pioneering papers on coherent states [22], where he realized first that "the amplitudes of successive coherent excitations of the mode add as complex numbers in quantum theory, just as they do in classical theory."

After this superposition, the photon-number distribution in the cavity has become

$p^{( \pm)}\left(n ; \alpha, \alpha_{r}\right)=\frac{1}{\mathcal{N}}\left|\left\langle n \mid \alpha+\alpha_{r}\right\rangle \pm\left\langle n \mid-\alpha+\alpha_{r}\right\rangle\right|^{2}$.

Let us consider separately the two quadratures. When $\alpha$ and $\alpha_{r}$ have the same phase, the photon distribution, which we will note $p_{(\text {in })}^{( \pm)}(n)$, appears as the sum of two quasi-Poissonian distributions peaked around $n=\left|\alpha+\alpha_{r}\right|^{2}$ and $\left|-\alpha+\alpha_{r}\right|^{2}$. The interference term in (58) is negligible provided the two Poisson distributions do not overlap, which is the case as soon as $\left|\alpha_{r}\right| \gg>1$. A particularly interesting situation arises when $\alpha$ and $\alpha_{r}$ have the same amplitude. $p_{(\text {in })}^{( \pm)}(n)$ then consists in a $\delta$ like peak at $n=0$ and a Poisson peak around $n=4|\alpha|^{2}$. After interfering with the $\alpha_{r}$ reference, the cavity field has become a linear superposition of the vacuum field with a classical field: the cavity is either "empty" or "filled" with a coherent field. Coupling a Schrödinger cat corresponding to two opposite phase fields having the same amplitude with a classical current thus results in creating a new Schrödinger cat, linear superposition of two states with different amplitudes. The important point for our purpose is to note that the existence of two separate peaks in the in-phase sum field is the proof of the existence of two classical fields in the cavity, prior to the injection of the reference. It does not prove, however, that these two fields are in a coherent quantummechanical superposition.

On the other hand, when $\alpha$ and $\alpha_{r}$ are $\pi / 2$ out of phase, $\alpha+\alpha_{r}$ and $-\alpha+\alpha_{r}$ are $c$ numbers with the same amplitude and the interference term in (58) is important. Assuming without loss of generality that $\alpha$ is real, we now define

$$
-\alpha \pm \alpha_{r}=\rho e^{ \pm i \theta}
$$

with

$$
\rho=\sqrt{|\alpha|^{2}+\left|\alpha_{r}\right|^{2}}
$$

and

$$
\tan \theta=\left|\alpha_{r}\right| /|\alpha|,
$$

and we get after a straightforward calculation,

$$
p_{\text {(out) }}^{( \pm)}(n)=\frac{2}{\mathcal{N}} e^{-\rho^{2}} \frac{\rho^{2 n}}{n !}(1 \pm \cos 2 n \theta)
$$

$p_{\text {(out) }}^{( \pm)}(n)$ exhibits a Poisson envelope with superimposed modulations, signaling the coherence between the two "classical" states. These modulations have opposite phases for the + and - cases. We have displayed in Figs. 12(a) and 12(b) the in-phase and out-of-phase photon-number distributions in the + case, obtained when $|\alpha|=\left|\alpha_{r}\right|=\sqrt{7}$ (7 photons in the initial coherent field and in the reference). 

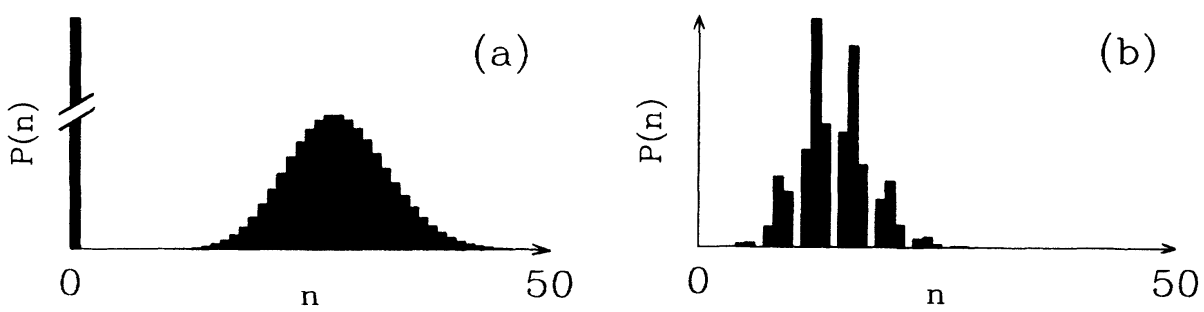

FIG. 12. Photon-number distributions obtained by superposing a reference field $\alpha_{r}$ to the $|\alpha\rangle+|-\alpha\rangle$ "Schrödinger cat" state $\left(|\alpha|=\left|\alpha_{r}\right|=\sqrt{7}\right)$. (a) $\alpha_{r}$ and $\alpha$ are in phase. The photon-number distribution is the sum of a $\delta$-like function at $n=0$ (height reduced to save space) and a Poisson distribution centered at $\bar{n}=28$. (b) $\alpha_{r}$ and $\alpha$ are $\pi / 2$ out of phase. The photon-number distribution, centered at $n=14$, exhibits oscillations with a period equal to 4 .

The $p_{(\text {in) }}^{( \pm)}(n)$ and $p_{(\text {out }}^{( \pm)}(n)$ distributions can actually be measured by detecting atoms sent through the cavity after injection of the reference, in an experiment quite similar to the one described in Sec. IV C. A complete sequence, including the preparation and the detection of the Schrödinger cats, would consist in four successive steps. (i) a coherent field $|\alpha\rangle$ is injected into the cavity; (ii) a first atom is sent through the first Ramsey zone, the cavity, and the second Ramsey zone, the fields in these zones and the atom velocity being adjusted as described in Sec. VI B. The atom is then detected in level $f$ or $e$, resulting in the preparation of one of the $\left|\Psi_{\epsilon=\pi}^{( \pm)}(\alpha)\right\rangle$ Schrödinger cat states. (iii) The reference beam $\left|\alpha_{r}\right\rangle$ is then injected into the cavity, either in or out of phase with $|\alpha\rangle$. (iv) A "train" of atoms with random velocities is then sent through the apparatus, their quantum states and velocities being measured downstream. A value of the photon number is obtained from this sequence. Statistics of a large number of such sequences corresponding to the same detection outcome for the first atom and the same phase choice for $\alpha_{r}$ allows us to reconstruct the $p_{(\text {in })}^{( \pm)}(n)$ and $p_{(\text {out })}^{( \pm)}(n)$ distributions. The presence of two peaks in the $p_{(\text {in })}^{( \pm)}(n)$ distribution, combined with oscillations in the $p_{(\text {out }}^{( \pm)}(n)$ one, is the unambiguous signature of "Schrödinger cat" state. We thus see that the phase properties of the Schrödinger cat states can be observed with the DAP-QND scheme, in spite of the fact that this method gives direct information only on diagonal matrix elements of the field. The trick consists in adding another field to the Schrödinger cat and detecting the corresponding interference effects, again on diagonal terms of the combined field density matrix.

The cavity must be allowed to relax between two measuring sequences, so that each measurement starts anew with the preparation of the same well-defined coherent field. On the other hand, the Schrödinger cat should not relax appreciably during steps (ii) and (iii) and negligible relaxation of the photon-number distribution should occur during step (iv) of each sequence. This imposes severe limits on the cavity $Q$ factor, since each of these steps should occur within a time much shorter than the characteristic field energy decay time $t_{\text {cav }}=Q / \omega$. We have seen indeed in Sec. IV D that the Fock states involved in the field photon-number distribution decay within a characteristic time $t_{\text {cav }} / \bar{n}$, which is the upper limit for the duration of step (iv). We will furthermore show below that the macroscopic quantum coherences of the Schrödinger cat decay also within the time $t_{\text {cav }} / \bar{n}$, which sets the same upper limit for steps (ii) and (iii).

\section{WIGNER REPRESENTATION}

The Wigner distribution is particularly convenient for displaying simultaneously the energy and phase information of a single mode field in a very simple and graphic form. It also allows for a simple analysis of the field relaxation. We rapidly recall here the Wigner representation formalism [34] and apply it to the study of the Schrödinger cat state "experiment."

\section{A. General properties of the Wigner distribution}

Let us recall some basic properties of the Wigner distribution of a field mode whose density operator is $\rho(t)$. We define first the field symmetric and normally ordered characteristic functions [34] as

$C_{S}(\lambda)=\left\langle\exp \left(\lambda a^{\dagger}-\lambda^{*} a\right)\right\rangle=\operatorname{Tr}\left[\rho \exp \left(\lambda a^{\dagger}-\lambda^{*} a\right)\right]$,

$$
\begin{aligned}
C_{\mathcal{N}}(\lambda) & =\left\langle\exp \left(\lambda a^{\dagger}\right) \exp \left(-\lambda^{*} a\right)\right\rangle \\
& =\operatorname{Tr}\left[\rho \exp \left(\lambda a^{\dagger}\right) \exp \left(-\lambda^{*} a\right)\right],
\end{aligned}
$$

where $\lambda$ is a $c$-number variable. $C_{S}(\lambda)$ and $C_{\mathcal{N}}(\lambda)$ are related by

$$
C_{S}(\lambda)=\exp \left(-\lambda^{2} / 2\right) C_{\mathcal{N}}(\lambda),
$$

which simply follows from a well-known theorem about the product of exponential operators.

The Wigner distribution $W(\alpha)$ of the complex amplitude $\alpha$ is the real two-dimensional Fourier transform of the symmetric characteristic function, defined as

$$
W(\alpha)=\frac{1}{\pi^{2}} \int \exp \left(-\lambda^{2} / 2\right) C_{\mathcal{N}}(\lambda) \exp \left(\alpha \lambda^{*}-\alpha^{*} \lambda\right) d^{2} \lambda
$$

The real and imaginary parts of $\alpha$ correspond to two quadrature amplitudes of the field $q$ and $p$ : 
$q=\left(\frac{h}{2 \omega}\right)^{1 / 2}\left(\alpha+\alpha^{*}\right), \quad p=\frac{1}{i}\left[\frac{\hbar \omega}{2}\right]^{1 / 2}\left(\alpha-\alpha^{*}\right)$.

$W(\alpha)$, considered as a function of $q$ and $p$, may be viewed as a "probability distribution" for the field quadrature components in phase space. Of course, $q$ and $p$ cannot be simultaneously determined with infinite precision due to uncertainty relations, so that $W(q, p)$ is only a quasidistribution which can take nonpositive values for limited ranges of $q$ and $p$.

It may be useful to express $W$ in terms of the matrix elements of $\rho$ in the photon-number representation:

$$
\begin{aligned}
& W(\alpha)=\frac{2}{\pi} e^{-2|\alpha|^{2}} \operatorname{Re}\left[\sum_{\substack{n, m=0 \\
n \geq m}}^{\infty}(-1)^{m}\left(2-\delta_{n m}\right)\left(\frac{m !}{n !}\right]^{1 / 2}\right. \\
&\left.\times(2 \alpha)^{n-m} \mathcal{L}_{m}^{n-m}\left(4|\alpha|^{2}\right) \rho_{m, n}\right],
\end{aligned}
$$

where

$\mathcal{L}_{m}^{n-m}(x)=\sum_{p=0}^{m}(-1)^{p} \frac{n !}{(n-m+p) !(m-p) ! p !} x^{p}$

is a Laguerre polynomial. Equation (68) is a generalization of a formula found in the literature for fields represented by a diagonal matrix in the Fock state basis $[35,26]$.

Finally, the Wigner representation is very convenient to study the relaxation of a field mode initially described by the density matrix $\rho(0)$. A straightforward calculation shows that if $\rho(t)$ evolves according to Eq. (42) with $n_{b}=0$ (relaxation produced by a $T=0 \mathrm{~K}$ reservoir), then $C_{\mathcal{N}}(\lambda, t)$ obeys the following differential equation [34]:

$\left[\frac{\partial}{\partial t}+\frac{1}{2 t_{\mathrm{cav}}}\left[\lambda \frac{\partial}{\partial \lambda}+\lambda^{*} \frac{\partial}{\partial \lambda^{*}}\right]\right] C_{\mathcal{N}}(\lambda, t)=0$,

whose solution is obtained by replacing $\rho$ by $\rho(0)$ and $\lambda$ by $\lambda e^{-t / 2 t}$ cav in Eq. (64). Using Eq. (66), we get

$$
\begin{aligned}
W(\alpha, t)=\int e^{-|\lambda|^{2} / 2} \operatorname{Tr}\left[\rho(0) \exp \left(\lambda e^{-t / 2 t_{\mathrm{cav}}} a^{\dagger}\right)\right. \\
\left.\times \exp \left(-\lambda^{*} e^{-t / 2 t_{\mathrm{cav}}} a\right)\right] \\
\times e^{\alpha \lambda^{*}-\alpha^{*} \lambda} d^{2} \lambda .
\end{aligned}
$$

\section{B. Wigner representations of special field states}

Simple analytical expressions for the $W$ distributions associated to states of particular interest in this study are easily obtained from the above equations, the corresponding distributions being conveniently represented in a three-dimensional $q, p, z$ space by the $z-W(q, p)=0$ surface. The $n$-Fock state Wigner distribution is directly deduced from (68):

$$
W(\alpha)=(-1)^{n} \frac{2}{\pi} e^{-2|\alpha|^{2}} \mathcal{L}_{n}^{(0)}\left(4|\alpha|^{2}\right)
$$

and is graphically represented in Fig. 13(a) for $n=5$. The

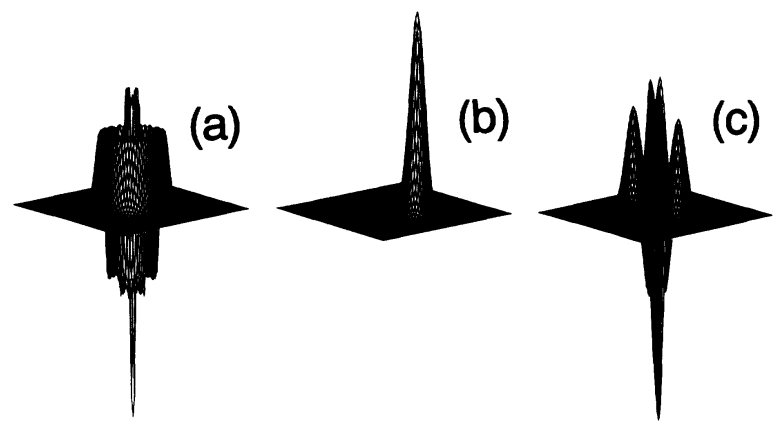

FIG. 13. Three-dimensional representations of Wigner distributions for special fields. $W$ is plotted along the vertical axis and the field quadratures $q$ and $p$ along the two orthogonal horizontal axes ("back" to "front" and left to right, respectively.) (a) Fock state $n=5$; (b) coherent state with $\bar{n}=5$; (c) Schrödinger cat state $\left|\Psi_{\epsilon}^{(+)}(\alpha)\right\rangle$ with $\alpha=\sqrt{5}$.

cylindrical symmetry of the Wigner surface corresponds to the lack of phase information in this state. The number of circular intersections of the surface with the $(q, p)$ plane corresponds to the number of photons in the state. From Eqs. (64) and (66) the Wigner distribution of a coherent state $\alpha_{0}$ is readily obtained by making use of the relation $a|\alpha\rangle=\alpha|\alpha\rangle$ :

$$
W(\alpha)=\frac{2}{\pi} e^{-2\left|\alpha-\alpha_{0}\right|^{2}} .
$$

It is represented by a Gaussian "bump" centered around $\alpha_{0}$ [Fig. 13(b)]. As for the Schrödinger cat states with the + or - sign [Eq. (56)], they are associated with the following distributions:

$$
\begin{aligned}
W_{ \pm}(\alpha)= & \frac{2}{\pi\left[1 \pm e^{-2\left|\alpha_{0}\right|^{2}}\right)} \\
& \times\left\{e^{-2\left|\alpha-\alpha_{0}\right|^{2}}+e^{-2\left|\alpha+\alpha_{0}\right|^{2}}\right. \\
& \left. \pm 2 e^{-2|\alpha|^{2}} \cos \left[4 \operatorname{Im}\left(\alpha \alpha_{0}^{*}\right)\right]\right\},
\end{aligned}
$$

whose expressions are also easily derived from Eqs. (64)-(66). The Wigner surface corresponding to the + sign Schrödinger cat is represented in Fig. 13(c). The first two Gaussians on the right-hand side of Eq. (74) correspond to the two "classical" fields, dephased by $\pi$ from one another. They are represented by two "bumps" symmetrical with respect to the origin. The third contribution on the right-hand side is an interference term, associated with the quantum coherence between the two states. It is a Gaussian centered around the origin, modulated by a cosine with a frequency of oscillation proportional to the amplitude of the field. The fringe lines $\left[\operatorname{Im}\left(\alpha \alpha_{0}^{*}\right)=\right.$ const] are parallel to the $\alpha_{0}$ direction, i.e., to the line joining the center of the two Gaussian "bumps." The - sign Schrödinger cat has the same distribution, with a $\pi$ shift in the phase of the fringe pattern. Wigner representation of Schrödinger cat states has been discussed in [30,31]. 


\section{Free relaxation of the Schrödinger cat state}

We briefly discuss here the evolution of a Schrödinger cat state relaxing freely in a cavity. Similar treatments of this problem can be found in [30,31]. We include this discussion here for sake of completeness, in view of the com- parison we make in the next section with a relaxing Schrödinger cat undergoing a continuous interaction with a beam of atoms.

Starting from the general Eq. (71), we easily find for the time evolution of a Schrödinger cat state subjected to relaxation at $T=0 \mathrm{~K}$ :

$$
\begin{aligned}
W_{ \pm}(\alpha, t)=\frac{2}{\pi\left(1 \pm e^{-2\left|\alpha_{0}\right|^{2}}\right)}\left\{\exp \left(-2\left|\alpha-\alpha_{0} e^{-t / 2 t_{\mathrm{cav}}}\right|^{2}\right)+\exp \left(-2\left|\alpha+\alpha_{0} e^{-t / 2 t_{\mathrm{cav}}}\right|^{2}\right)\right. \\
\left. \pm 2 e^{-2|\alpha|^{2}} \cos \left[4 \operatorname{Im}\left(\alpha \alpha_{0}^{*}\right) e^{-t / 2 t_{\mathrm{cav}}}\right] \exp \left[-2\left|\alpha_{0}\right|^{2}\left(1-e^{-t / 2 t_{\mathrm{cav}}}\right)\right]\right\} .
\end{aligned}
$$

When $t=0$ this expression reduces to (74). For $t<<t_{\text {cav }}$, we see that the central peak, associated with the coherence between the two states, decays as $\exp \left(-2\left|\alpha_{0}\right|^{2} t / t_{\text {cav }}\right)$. The rate of decay of the coherence is therefore proportional to the intensity of the initial field in the cavity. At the same time, the two other peaks approach the origin with a rate equal to $1 / 2 t_{\text {cav }}$. As $t \rightarrow \infty$, the distribution (75) approaches a single Gaussian around the origin, which is just the Wigner distribution for the vacuum state. The relaxation of the Schrödinger cat state is shown in Fig. 14, where the Wigner distribution is plotted at various times following the preparation of the state (to save space, only the positive part of $W$ is shown). The initial state is a coherent superposition of two opposite classical fields with $n=\left|\alpha_{0}\right|^{2}=5$ [Fig. 14(a)]. We notice that after a time of the order of $t_{\text {cav }} / 10$, the central feature of the distribution signaling the Schrödinger cat's coherence has appreciably decreased while the position of the two bumps has practically remained unchanged [Fig. 14(b)]. After a time $t_{\text {cav }} / 3$, the distribution has evolved into a nearly incoherent superposition of two classical fields [Fig. 14(d)], which is to a good approximation represented by the density matrix

$$
\rho=\frac{1}{2}\left(\left|\alpha_{t}\right\rangle\left\langle\alpha_{t}|+|-\alpha_{t}\right\rangle\left\langle-\alpha_{t}\right|\right)
$$

$$
\text { with } \alpha_{t}=\alpha_{0} e^{-t / 2 t_{\mathrm{cav}}} \text {. }
$$

Finally, the distribution turns into the one corresponding to the vacuum state [Fig. 14(h)]. The fast decay of the Schrödinger cat under the effect of relaxation is a well-known feature of macroscopic quantum coherences. It restricts the practical observation of such coherences to relatively small systems for which $n$ is not too large. The Schrödinger cat is generated in a time $L_{c} / v$, of the order of $10^{-3} \mathrm{~s}$. The Schrödinger cat's detection experiment involves the injection of a reference field $\alpha_{r}$ in the cavity, which can be performed in a time of the order of $10^{-3} \mathrm{~s}$, or even shorter. If the cavity-damping time is of the order of $0.1 \mathrm{~s}$, a Schrödinger cat state with $n$ as large as 100 could be generated and observed. These values of $n$ are markedly higher than those in other proposals of experimental realizations of optical Schrödinger cats.

\section{Evolution of Wigner distribution under continuous interaction with atoms}

We finally discuss the effect of continuous monitoring on a relaxing Schrödinger cat. We assume as in Sec. VI that the field interacts with a beam of monokinetic atoms, each of which imparts, when in level $e$, an $\epsilon=\pi$ phase shift to the field. The first atom prepares an initial Schrödinger cat state, for example the even-photon-

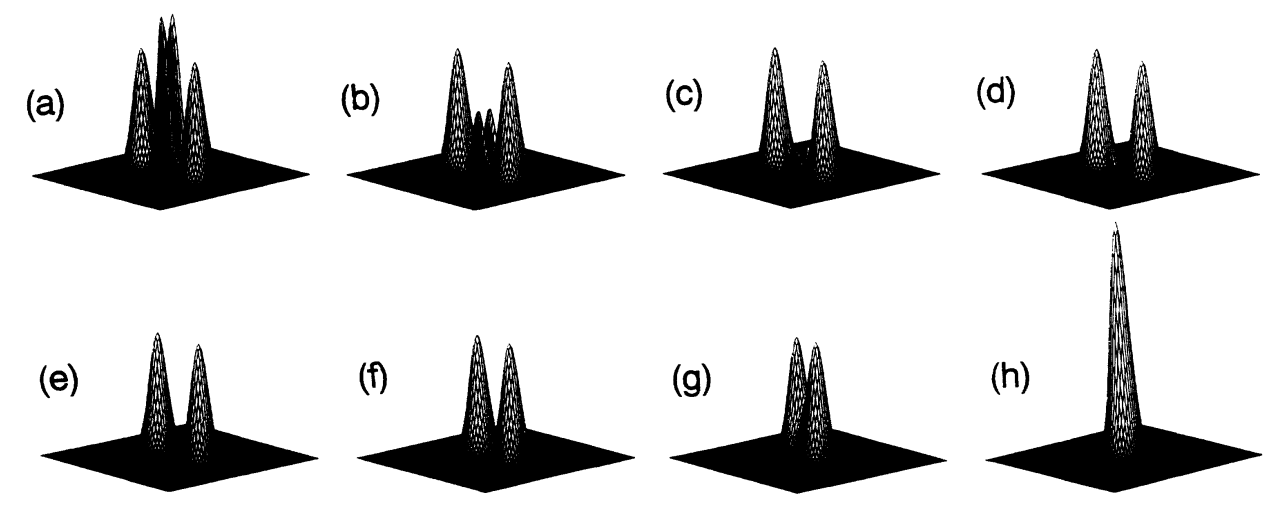

FIG. 14. Evolution of the positive part of the Wigner distribution of a freely relaxing Schrödinger cat state $(\alpha=\sqrt{5})$. The quantities being plotted along the three axes are the same as in Fig. 13. (a) Initial field at $t=0$; (b) - (h) field at times $t=t_{\text {cav }} / 10, t=t_{\text {cav }} / 5$, $t=t_{\mathrm{cav}} / 3, t=t_{\mathrm{cav}} / 2, t=t_{\mathrm{cav}}, t=2 t_{\mathrm{cav}}$, and $t=\infty$. 
number one. As this cat relaxes in the cavity, it continuously interacts with subsequent atoms, all of which are supposed to be detected. The simulation of this experiment is straightforward. We use in the computation the Fock state representation of the density matrix, with Eqs. (35) to determine the evolution of the density matrix induced by measurements and Eq. (43) to describe field relaxation between consecutive atoms. Equation (68) is then used to get the Wigner distribution of the field at any time. Figure 15 shows the evolution of this Wigner distribution for one realization of the simulation. The initial field is again a classical one with $\bar{n}=5$. The atoms are supposed to cross the cavity at a regular rate, the cavity relaxation time corresponding to 50 atoms. Several features are different from the ones displayed in Fig. 14. The most striking is the fact that the Schrödinger cat's coherence survives much longer, practically until the last stage of the field decay. Note also that, from time to time (after atom number $3,6,14, \ldots$ in this particular realization), the parity of the Schrödinger cat abruptly switches from + to - or from - to + (this is revealed by the change of the phase of the interfering pattern in the Wigner distribution central feature). These results are easy to explain qualitatively. Immediately after the first atom detection (in level $f$ in this particular realization), the odd photon numbers have been completely suppressed. Between the first and the second atom, the relaxation had the effect of slightly repopulating these photon numbers. The probability of detecting the second atom in level $e$ [given by Eq. (54b)] was thus not zero, though it remained very small. This atom therefore had a probability close to unity of being detected in the same level $f$ as the first one, which has actually been the outcome of this realization. As a result, the odd-photonnumber states have been suppressed again and the initial Schrödinger cat state restored.
Since relaxation slightly populates the other parity states between detection events, an atom is eventually detected in the other level (atom number 3 in the realization shown in Fig. 15). Immediately after this event, the field "jumps" into the "- " cat state, until another event of the same kind brings it back to the "+" state (after atom number 6 in this realization), and so on. In other words, each detected atom refreshes the coherence between the two classical fields by destroying photon numbers of a given parity. Most often, it maintains the Schrödinger cat's parity, but from time to time it induces it to "jump" from one parity to the other as the photon number suddenly switches from even to odd or back. These measurement-induced effects superimpose to the classical field relaxation, which, at the rate $1 / t_{\text {cav }}$, brings the field down to the vacuum state. Here again, the behavior of a "freely" decaying Schrödinger cat is reproduced by statistical averaging of a large number of such realizations. Since the precise time of a jump between + and - states is random, such an averaging kills very fast the macroscopic coherence between the two classical components and the results shown in Fig. 14 are recovered.

The mechanism by which an atom measurement process refreshes the statistical "purity" of a quantum field is easy to understand in another way. Assume that the second atom is sent through the system after a time of the order of $t_{\text {cav }}$ so that the initial Schrödinger cat has decayed to the statistical mixture described by Eq. (76). This second atom has then a $50 \%$ probability of being detected in either state $e$ or $f$. If it is actually observed in $f$, each of the two components $\alpha_{t}$ and $-\alpha_{t}$ of the statistical mixture (76) are transformed into the same state $\left|\alpha_{t}\right\rangle+\left|-\alpha_{t}\right\rangle$. As a result, the statistical mixture has been turned into a pure state Schrödinger cat again. The same is true if the atom is observed in level $e$ (the
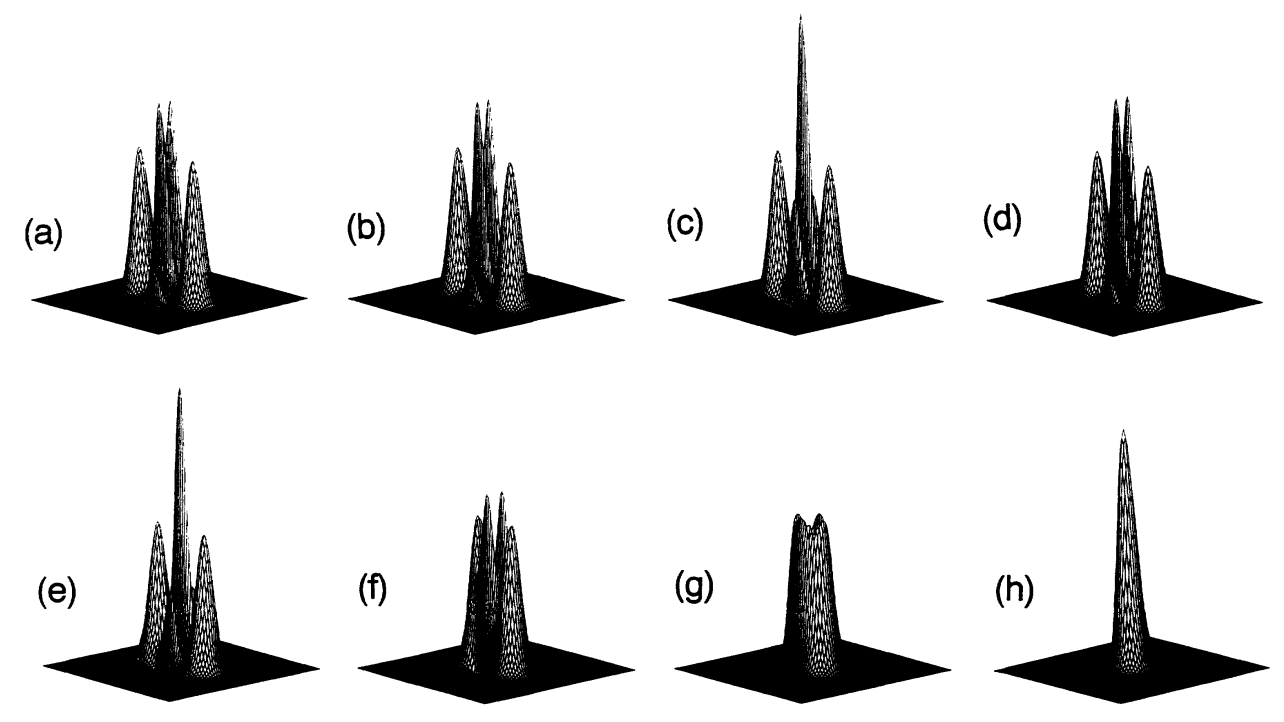

FIG. 15. Evolution of the positive part of the Wigner distribution of a relaxing Schrödinger cat continuously interacting with a monokinetic beam of atoms $(\epsilon=\pi)$. The quantities being plotted against the three axes are the same as in Fig. 13. (a)-(g) Fields after $1,2,3,6,14,50,100$, atoms, respectively; (h) field at $t=\infty$. Atoms are supposed to be regularly injected in the cavity and all detected. $t_{\text {cav }}$ corresponds to the passage of 50 atoms. 
Schrödinger cat $\left|\alpha_{t}\right\rangle-\left|-\alpha_{t}\right\rangle$ then being created). It may at first sight seem paradoxical that an irreversible collapse of the system wave function induced by the atom measurement restores "coherences" in the system. The paradox comes from the fact that "coherence" has several meanings in this problem. The macroscopic coherence between the two classical fields has been reestablished, but no coherence between Fock states has been created in the process. It should be noted indeed that all quantum coherences are not killed at the rate $\bar{n} / 2 t_{\text {cav }}$ by the relaxation process. In fact, the "incoherent" superposition of Eq. (76) corresponds to an ordinary Poisson distribution for the photon number with all $\rho_{n, n^{\prime}}$ coherences corresponding to $n-n^{\prime}$ odd suppressed, while all the coherences $\rho_{n, n^{\prime}}$ associated to $n-n^{\prime}$ even are still large. The effect on this field of an atom measurement in level $f$ is to kill the odd photon numbers in the distribution, without affecting the $n-n^{\prime}$ even coherences. The result is to recreate an even-photon-number Schrödinger cat, without actually producing any $\rho_{n, n^{\prime}}$ coherence which was not already present in the field.

\section{CONCLUDING REMARKS}

The experiments proposed in this article link together the concepts of cavity quantum electrodynamics and atomic interferometry, two rapidly expanding domains in quantum optics. We have shown that a field stored in a confined space can be continuously monitored and manipulated by detecting interferences on nonresonant atoms coupled to that field. No energy is exchanged with the field, whose state is modified only through an information-gathering process.

In our previous work [5] and in this paper we have chosen to make use of the Ramsey fringes technique in order to produce and detect the atomic interferences. The method can obviously be generalized to other interference schemes. In an atomic Young's double-slit experiment [36] a cavity containing the field to be measured could be placed in front of one of the slits, resulting in an $n$-dependent shift of the fringe pattern, whose detection would provide the same information as the one obtained from the Ramsey pattern described here. Another variant, proposed in [37], consists in detecting the dipoleforce-induced Bragg scattering of an atomic beam on a standing-wave cavity mode. Also somewhat related to the present work are the recent proposals which suggest performing QND measurements of photons by detecting electron interferences in semiconductor microstructures [38] or in Aharonov-Bohm interfering designs [39].

The Rydberg atom-microwave cavities combination has many attractive features. One of its assets is the very long cavity-damping time, permitting us to gather the information and to manipulate the field before it decays. In this respect, the experiments proposed here appear as "photon trap" experiments, "dual" of the ion trap experiments in which material particles are manipulated by means of fields. Realization of these experiments would constitute a paradigm of measurement, testing the basic postulates of quantum mechanics. A variety of "gedanken experiments" on a quantum field become possible: demonstration of photon jumps, generation of coherences between macroscopically different states, inhibition of field-coherent evolution under continuous observation (quantum Zeno effect).

For these experiments, it is important to be able to estimate in detail the effect of the imperfections of a real experiment. Our system being entirely calculable, the influence of velocity measurement uncertainty, finite contrast of the fringe signal, and noise of the atomic detection counters, can be included in the numerical simulations in a detailed way. This discussion is planned to be published in a forthcoming paper.

\section{ACKNOWLEDGMENTS}

Two of the authors (L.D. and N.Z.) are thankful to Ecole Normale Supérieure for its hospitality, and to the Centre National de la Recherche Scientifique (CNRS) and Conselho Nacional de Desenvolvimento Cientifico e Tecnologico $(\mathrm{CNPq})$ for their financial support.

\section{APPENDIX}

We derive here the expressions of the $b_{a}\left(n, v ; \varphi_{0}, \epsilon\right)$ amplitudes. We assume that the cavity field contains $n$ photons. In the interaction representation with respect to the unperturbed atomic and cavity field Hamiltonians, the atom plus field system wave function can be expanded as

$\left|\Psi^{(\text {atom }+ \text { field })}\right\rangle=b_{e}(n, t)|e, n\rangle+b_{f}(n, t)|f, n\rangle$,

where $b_{e}(n, t)$ and $b_{f}(n, t)$ are time-dependent functions whose evolution is due only to the effects of the auxiliary fields in $R_{1}$ and $R_{2}$ and to the dispersive interaction between the atom and the field in the cavity. The initial conditions for these amplitudes, before the atom enters in zone $R_{1}$, are

$$
b_{e}(n)=1, \quad b_{f}(n)=0 .
$$

We assume for the sake of simplicity that the auxiliary field is uniform in $R_{1}$ and $R_{2}$ with a Rabi frequency $\Omega_{r}$. We call $\delta L$ the length of each zone, which is crossed by an atom with velocity $v$ in a time $\tau=\delta L / v$. We also suppose that the detuning $\omega_{r}-\omega_{e f}$ is small compared to $1 / \tau$, so that it can be neglected in the equations describing the atom evolution in the two Ramsey zones.

During the time the atom crosses $R_{1}$, the amplitudes $b_{a}(n, t)$ evolve according to the coupled equations:

$$
\dot{b}_{f}(n, t)=-\frac{\Omega_{r}}{2} b_{e}(n, t), \quad \dot{b}_{e}(n, t)=\frac{\Omega_{r}}{2} b_{f}(n, t)
$$

which, taking into account the boundary conditions (A2), admit the following solutions at time $\tau$ :

$$
b_{e}(n)=\cos \frac{\Omega_{r} \tau}{2}, \quad b_{f}(n)=-\sin \frac{\Omega_{r} \tau}{2} .
$$

After the atom has crossed the cavity containing $n$ photons, $b_{e}$ has been phase shifted while $b_{f}$ has remained unchanged. The initial conditions for the atomic amplitudes before the atom enters zone $R_{2}$ are thus 
$b_{e}(n)=\cos \frac{\Omega_{r} \tau}{2} e^{-i n \epsilon v_{0} / v}, \quad b_{f}(n)=-\sin \frac{\Omega_{r} \tau}{2}$.

In the second zone, these amplitudes evolve according to

$$
\begin{aligned}
& \dot{b}_{f}(n, t)=-\frac{\Omega_{r}}{2} e^{i \varphi_{0} v_{0} / v} b_{e}(n, t), \\
& \dot{b}_{e}(n, t)=\frac{\Omega_{r}}{2} e^{-i \varphi_{0} v_{0} / v} b_{f}(n, t) .
\end{aligned}
$$

These equations differ from Eq. (A3) by complex exponential factors describing the phase shift between the auxiliary field and the atomic coherence during the flight time of the atom between the two zones. The evolution of these amplitudes during a time interval $\tau$ form their initial values given by Eq. (A5) is straightforward to compute. After zone $R_{2}$, the system state is described by

$$
\begin{aligned}
& b_{e}(n)=\cos ^{2} \frac{\Omega_{r} \tau}{2} e^{-i n \epsilon v_{0} / v}-\sin ^{2} \frac{\Omega_{r} \tau}{2} e^{-i \varphi_{0} v_{0} / v}, \\
& b_{f}(n)=-\frac{1}{2} \sin \Omega_{r} \tau\left(1+e^{i\left(\varphi_{0}-n \epsilon\right) v_{0} / v}\right) .
\end{aligned}
$$

We finally assume that $\Omega_{r} \tau=\pi / 2$ when the atomic velocity is $v_{0}$ and we immediately obtain expressions (24a) and (24b) for the final amplitudes $b_{a}$. Of course, these amplitudes depend explicitly upon the arguments $n, v, \varphi_{0}$, and $\epsilon$.
[1] V. B. Braginsky and F. I. Khalili, Zh. Eksp. Teor. Fiz. 78, 1712 (1980) [Sov. Phys. JETP 51, 859 (1980)]; G. J. Milburn and D. F. Walls, Phys. Rev. A 28, 2065 (1980).

[2] N. Imoto, H. A. Haus, and Y. Yamamoto, Phys. Rev. A 32, 2287 (1985).

[3] M. D. Levenson, R. M. Shelby, M. Reid, and D. F. Walls, Phys. Rev. Lett. 57, 2473 (1986); N. Imoto, S. Watkins, and Y. Sasaki, Opt. Commun. 61, 159 (1987); A. LaPorta, R. E. Slusher, and B. Yurke, Phys. Rev. Lett. 62, 28 (1989); P. Grangier, J. F. Roch, and G. Roger, ibid. 66, 1418 (1991).

[4] V. B. Braginsky, Y. I. Vorontsov, and F. I. Khalili, Zh. Eksp. Teor. Fiz. 73, 1340 (1977) [Sov. Phys. JETP 46, 705 (1977)]; W. G. Unruh, Phys. Rev. D 18, 1764 (1978).

[5] M. Brune, S. Haroche, V. Lefêvre, J. M. Raimond, and N. Zagury, Phys. Rev. Lett. 65, 976 (1990).

[6] S. Haroche, M. Brune, and J. M. Raimond, in Atomic Physics 12, Proceedings of the 12th International Conference on Atomic Physics, Ann Arbor, 1990, edited by R. R. Lewis and J. C. Zorn (American Institute of Physics, New York, in press); M. Brune, J. M. Raimond, S. Haroche, and L. Davidovich, in Laser Spectroscopy $X$, Proceedings of the 10th International Conference on Laser Spectroscopy, edited by M. Ducloy, E. Giacobino, and G. Camy (World Scientific, Singapore, in press).

[7] E. Schrödinger, Naturwissenschaften 23, 807 (1935); 23, 823 (1935); 23, 844 (1935) [English translation by J. D. Trimmer, Proc. Am. Phys. Soc. 124, 3325 (1980)].

[8] B. Yurke and D. Stoler, Phys. Rev. Lett. 57, 13 (1986); G. J. Milburn, Phys. Rev. A 33, 674 (1986); G. J. Milburn and C. A. Holmes, Phys. Rev. Lett. 56, 2237 (1986).

[9] D. J. Wineland and W. M. Itano, Phys. Today 40(6), 34 (1987).

[10] W. Nagourney, J. Sandberg, and H. Dehmelt, Phys. Rev. Lett. 56, 2797 (1986); T. Sauter, W. Neuhauser, R. Blatt, and P. Toschek, ibid. 56, 1696 (1986); J. C. Bergquist, R. G. Hulet, W. M. Itano, and D. J. Wineland, Phys. Rev. Lett. 57, 1699 (1986).

[11] W. M. Itano, D. J. Heinzen, J. J. Bollinger, and D. J. Wineland, Phys. Rev. A 41, 2295 (1990).

[12] N. F. Ramsey, Molecular Beams (Oxford University Press, New York, 1985).

[13] C. M. Caves, K. S. Thorne, R. W. D. Drever, V. D. Sandberg, and M. Zimmerman, Rev. Mod. Phys. 52, 341 (1980).

[14] S. Haroche, in New Trends in Atomic Physics, Les Houches
Summer School Session XXXVIII, edited by G. Grynberg and R. Stora (North-Holland, Amsterdam, 1984); S. Haroche and J. M. Raimond, in Advances in Atomic and Molecular Physics Vol. XX, edited by D. R. Bates and B. Bederson (Academic, New York, 1985).

[15] S. Haroche, in Fundamental Systems in Quantum Optics, Les Houches Summer School Session LIII, edited by J. Dalibard, J. M. Raimond, and J. Zinn-Justin (NorthHolland, Amsterdam, in press).

[16] F. Bernardot, P. Nussenzveig, M. Brune, J. M. Raimond, and S. Haroche, Europhys. Lett. 17, 33 (1992).

[17] C. Cohen-Tannoudji, Ann. Phys. (Paris) 7, 423 (1962); 7, 469 (1962).

[18] D. Meschede, H. Walther, and G. Müller, Phys. Rev. Lett. 54, 551 (1985); M. Brune, J. M. Raimond, P. Goy, L. Davidovich, and S. Haroche, ibid. 59, 1899 (1987).

[19] R. G. Hulet and D. Kleppner, Phys. Rev. Lett. 51, 1430 (1983); A. Nussenzveig, J. Hare, A. M. Steinberg, L. Moi, M. Gross, and S. Haroche, Europhys. Lett. 14, 755 (1991).

[20] S. Chu and C. Weiman (feature editors), J. Opt. Soc. Am. B 6, 2019 (1989).

[21] H. Paul, Quantum Opt. 3, 169 (1991).

[22] R. J. Glauber, Phys. Rev. 131, 2766 (1963).

[23] P. Meystre and E. M. Wright, Phys. Rev. A 37, 2524 (1988); P. Meystre, Opt. Lett. 12, 669 (1987).

[24] N. Imoto, M. Ueda, and T. Ogawa, Phys. Rev. A 41, 4127 (1990).

[25] Ning Lu, Phys. Rev. A 40, 1707 (1989).

[26] J. Jansky and T. Kobayashi, Phys. Rev. A 41, 4074 (1990).

[27] G. Rempe, F. Schmidt-Kaler, and H. Walther, Phys. Rev. Lett. 64, 2783 (1990).

[28] D. T. Pegg and S. M. Barnett, Phys. Rev. A 39, 1665 (1989); S. M. Barnett and D. T. Pegg, ibid. 41, 3427 (1990).

[29] P. Anantha and S. Swain, Phys. Rev. A 42, 5632 (1990).

[30] A. Vidiella Barranco, V. Buzek, P. L. Knight, and W. K. Lai, in Quantum Measurements in Optics, edited by $\mathrm{P}$. Tombesi (Plenum, New York, 1991); V. Buzek, A. Vidiella Barranco, and P. L. Knight (unpublished).

[31] W. Schleich, M. Pernigo, and F. Le Kien, Phys. Rev. A 44, 2172 (1991).

[32] B. Yurke, W. Schleich, and D. F. Walls, Phys. Rev. A 42, 1703 (1990).

[33] T. Ogawa, M. Ueda, and N. Imoto, Phys. Rev. A 43, 6458 (1991).

[34] J. Perina, Quantum Statistics of Linear and Non Linear 
Optical Phenomena (Reidel, Dordrecht, 1984).

[35] R. J. Glauber, in Quantum Optics and Electronics, Les Houches 1964 Summer School, edited by C. DeWitt, A. Blandin, and C. Cohen-Tannoudji (Gordon and Breach, New York, 1965).
[36] O. Carnal and J. Mlynek, Phys. Rev. Lett. 66, 2689 (1991).

[37] M. J. Holland, D. F. Walls, and P. Zoller, Phys. Rev. Lett. 67, 1716 (1991).

[38] A. Shimizu, Phys. Rev. A 43, 3819 (1991).

[39] M. D. Levenson, Phys. Rev. A 42, 2935 (1990). 


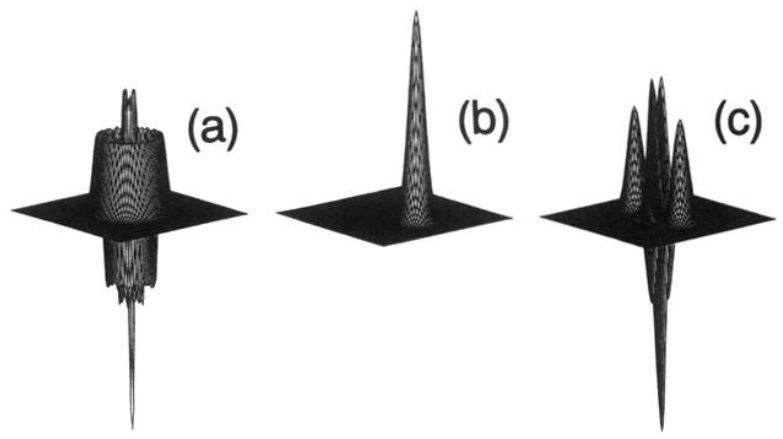

FIG. 13. Three-dimensional representations of Wigner distributions for special fields. $W$ is plotted along the vertical axis and the field quadratures $q$ and $p$ along the two orthogonal horizontal axes ("back" to "front" and left to right, respectively.) (a) Fock state $n=5$; (b) coherent state with $\bar{n}=5$; (c) Schrödinger cat state $\left|\Psi_{\epsilon}^{(+)}(\alpha)\right\rangle$ with $\alpha=\sqrt{5}$. 

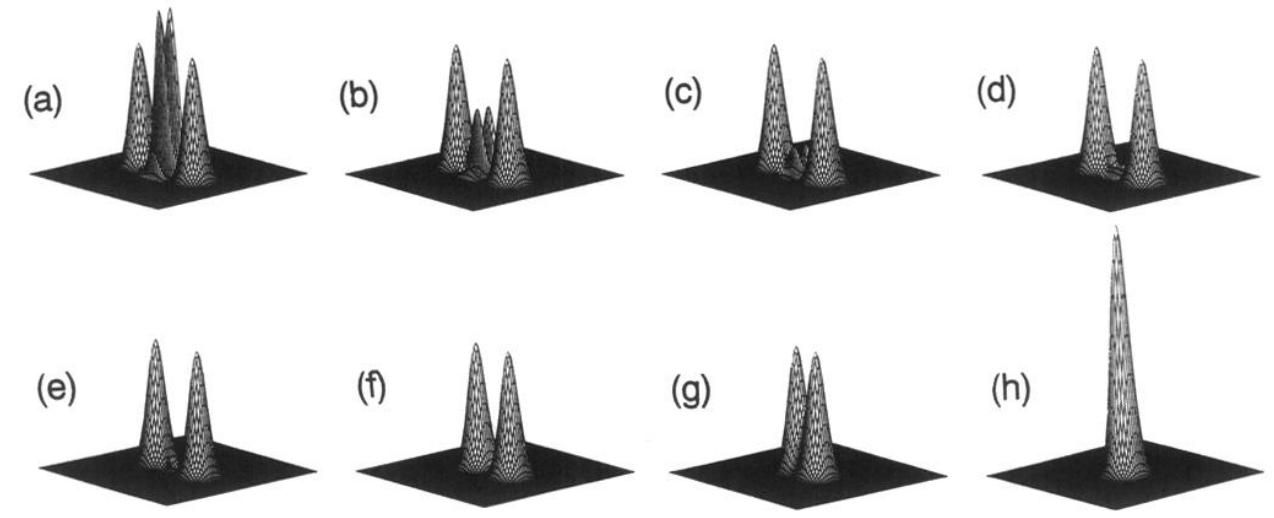

FIG. 14. Evolution of the positive part of the Wigner distribution of a freely relaxing Schrödinger cat state $(\alpha=\sqrt{5})$. The quantities being plotted along the three axes are the same as in Fig. 13. (a) Initial field at $t=0$; (b) - (h) field at times $t=t_{\text {cav }} / 10, t=t_{\text {cav }} / 5$, $t=t_{\text {cav }} / 3, t=t_{\text {cav }} / 2, t=t_{\text {cav }}, t=2 t_{\text {cav }}$, and $t=\infty$. 


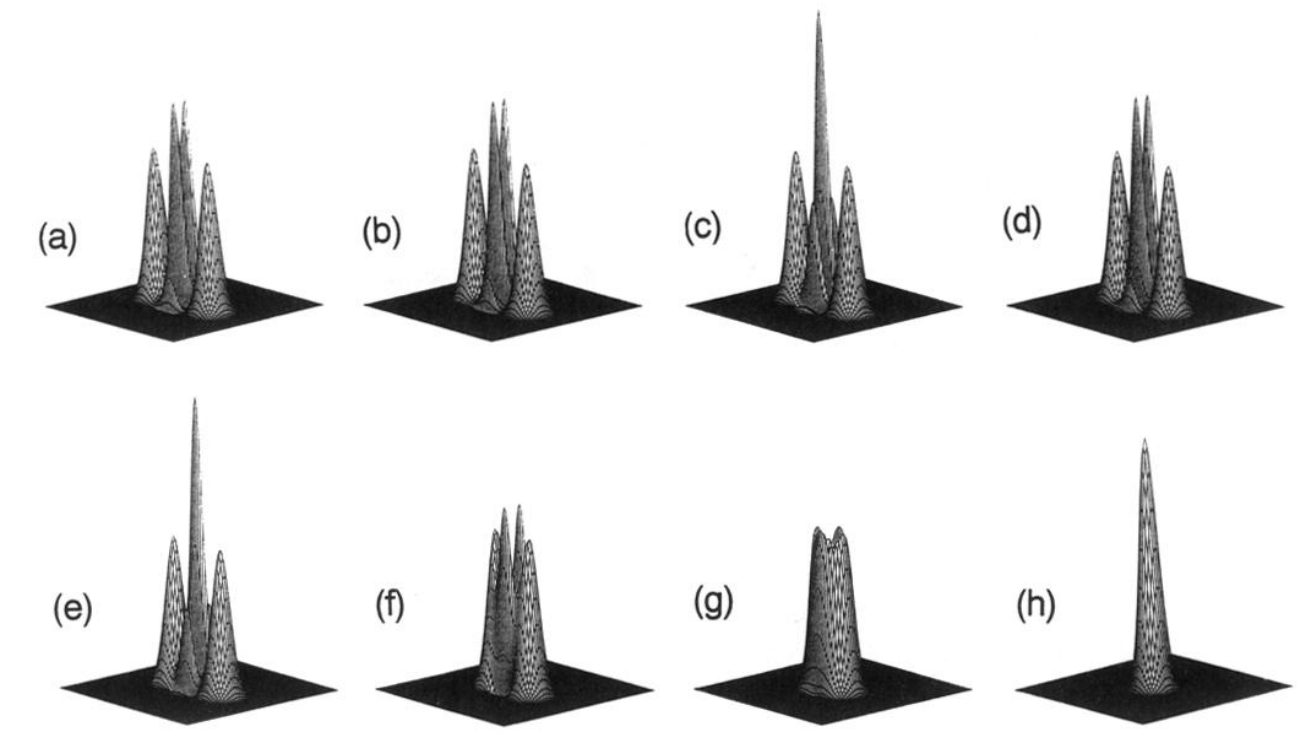

FIG. 15. Evolution of the positive part of the Wigner distribution of a relaxing Schrödinger cat continuously interacting with a monokinetic beam of atoms $(\epsilon=\pi)$. The quantities being plotted against the three axes are the same as in Fig. 13. (a)-(g) Fields after $1,2,3,6,14,50,100$, atoms, respectively; (h) field at $t=\infty$. Atoms are supposed to be regularly injected in the cavity and all detected. $t_{\text {cav }}$ corresponds to the passage of 50 atoms. 